\title{
Einstein manifolds with convex boundaries
}

\author{
Jean-Marc Schlenker
}

\begin{abstract}
Let $(\mathrm{M}, \partial \mathrm{M})$ be a compact $m+1$-manifold with boundary with an Einstein metric $g_{0}$, with ric $_{g_{0}}=-m g_{0}$ and with pinched negative curvature, such that $\partial \mathrm{M}$ is convex and umbilical. Let $h_{0}$ be the induced metric on $\partial \mathrm{M}$. Then any metric close enough to $h_{0}$ is induced on $\partial \mathrm{M}$ by an Einstein metric $g$ with $\operatorname{ric}_{g}=-m g$ on $\mathrm{M}$. A similar (but slightly weaker) result applies to Ricci-flat manifolds.

Résumé. Soit $(\mathrm{M}, \partial \mathrm{M})$ une $m+1$-variété compacte à bord, munie d'une métrique d'Einstein $g_{0}$, avec ric $_{g_{0}}=-m g_{0}$ et à courbure négative pincée, telle que $\partial \mathrm{M}$ est convexe et ombilique. Soit $h_{0}$ la métrique induite sur $\partial \mathrm{M}$. Alors toute métrique suffisamment proche de $h_{0}$ est induite sur $\partial \mathrm{M}$ par une métrique d'Einstein $g$ avec $\operatorname{ric}_{g}=-m g$ sur $\mathrm{M}$. Un résultat similaire (un peu plus faible) s'applique aux variétés Ricci-plates.
\end{abstract}

Mathematics Subject Classification (2000). 53C25; 53C45.

Keywords. Einstein manifolds; convex; boundary; umbilic; surface; isometric embeddings.

\section{Introduction}

A well-known theorem of Nirenberg [Nir53] asserts that any smooth metric on $\mathrm{S}^{2}$ with curvature $K>0$ admits a unique smooth isometric embedding in $\mathbf{R}^{3}$. An analogous result was proved by Aleksandrov [Ale58] and Pogorelov [Pog73] (see [Lab89] for a modern proof and more) in the hyperbolic space: any smooth metric on $\mathrm{S}^{2}$ with curvature $\mathrm{K}>-1$ admits a unique isometric embedding into $H^{3}$. Since those embedded spheres are convex and bound a ball, those results can be reformulated as follows:

Theorem 1. [Nirenberg, Aleksandrov, Pogorelov] Let $\mathrm{K}_{0} \in\{-1,0\}$. For any smooth metric $h$ on $\partial \mathrm{B}^{3}$ with curvature $\mathrm{K}>\mathrm{K}_{0}$, there exists a unique smooth metric $g$ on $\mathrm{B}^{3}$ with constant curvature $\mathrm{K}_{0}$ which induces $h$ on $\mathrm{B}^{3}$.

The "modern" proof of this theorem has 3 parts:

1. the operator $\phi$ sending a convex embedding to its induced metric is Fredholm (with index 0) at each (strictly) convex embedding; 
2. $\mathrm{T} \phi$ is injective at each of those points, i.e. convex surfaces are rigid;

3. $\phi$ is proper.

It is then possible to apply the Nash-Moser inverse function theorem to obtain that $\phi$ is a bijection between the relevant spaces of immersions and of metrics.

The hyperbolic version of theorem 1 has been extended by Labourie [Lab92], partially solving a conjecture of Thurston:

Theorem 2. [Labourie] Let ( $\mathrm{M}, \partial \mathrm{M})$ be a compact 3-manifold with boundary which admits a complete convex co-compact hyperbolic metric. Any metric $h$ on $\partial \mathrm{M}$ with curvature $\mathrm{K}>-1$ is induced by a hyperbolic metric $g$ on $\mathrm{M}$.

The uniqueness of $g$ was also conjectured by Thurston, but is still unknown (at least to the author).

The main goal of this paper is to give a partial extension of theorems 1 and 2 to higher dimensions. For dimensional reasons, it is not possible to do so in the setting of constant curvature spaces; we will try, given a metric on $\partial \mathrm{M}$, to show that it is induced on $\partial \mathrm{M}$ by an Einstein metric on $\mathrm{M}$. This makes sense since, in dimension 3, the Einstein metrics are just the constant curvature metrics.

We are not able, however, to obtain a global existence and uniqueness result as in theorem 1. This is because we only have the equivalent of point (1) of the sketch of proof above, and partially of point (2) since we prove the rigidity result we need only for metrics close to one for which $\partial \mathrm{M}$ is umbilical.

In the whole paper, $(\mathrm{M}, \partial \mathrm{M})$ is a compact, $\mathrm{C}^{\infty}(m+1)$-manifold with boundary $(m \geq 2)$. Since we will always assume that $\mathrm{M}$ admits an Einstein metric, it will be implicit that $\mathrm{M}$ is in fact analytic. In the negatively curved case, we have:

Theorem 3. Let $g_{0}$ be an Einstein metric on $\mathrm{M}$, with ric $_{g_{0}}=m \mathrm{~K}_{0} g_{0}, \mathrm{~K}_{0}<0$. Suppose that $\partial \mathrm{M}$ is strictly convex, umbilical, with a $\mathrm{C}^{\infty}$ induced metric $h_{0}$ and that, at each point:

$$
\mathrm{K}_{\max } \leq-\frac{2 m\left(-\mathrm{K}_{0}\right)}{3 m+1} \text { or } \mathrm{K}_{\min } \geq-\frac{4 m\left(-\mathrm{K}_{0}\right)}{3 m+1}
$$

Then there exists a neighborhood $U_{0}$ of $h_{0}$ in the space of $\mathrm{C}^{\infty}$ metrics on $\partial \mathrm{M}$ such that, for each $h \in U_{0}$, there exists an Einstein metric $g$ on $\mathrm{M}$ (with ric $_{g}=$ $m \mathrm{~K}_{0} g$ ) inducing $h$ on $\partial \mathrm{M}$.

Here $\mathrm{K}_{\min }$ and $\mathrm{K}_{\max }$ are, at each point of $\mathrm{M}$, the minimum and the maximum of the sectional curvatures of $g_{0}$. Equation (1) can be considered as a pinching condition because of the additional hypothesis that $g_{0}$ is Einstein. (1) means that $g_{0}$ can not be "too far" from having constant curvature. This is necessary to obtain a local rigidity result which is crucial in the proof.

Technically, the following condition is necessary. Call $\stackrel{\circ}{\mathrm{R}}_{g_{0}}$ the curvature operator acting on symmetric 2-tensor, and let $a_{0}$ be the highest eigenvalue of its 
restriction to trace-free symmetric 2-tensors. It is necessary that:

$$
(3 m+1) a_{0}+\mathrm{K}_{0} m(m+3) \leq 0 .
$$

This makes sense since (see [Bes87], 12.70) $\stackrel{\circ}{\mathrm{R}}_{g_{0}}$ preserves trace-free symmetric 2-tensors. It is proved in [Bes87], 12.71 that:

$$
a_{0} \leq \min \left\{(m-1) \mathrm{K}_{\max }-m \mathrm{~K}_{0}, m \mathrm{~K}_{0}-(m+1) \mathrm{K}_{\min }\right\},
$$

where $\mathrm{K}_{\min }, \mathrm{K}_{\max }$ are the minimum and maximum of the sectional curvature of $g$. This shows that (1) implies (2).

The proof actually shows a little more: $g$ is "locally unique", i.e. the operator $\partial$ sending an Einstein metric on $M$ to the induced metric on the boundary is a bijection from a neighborhood of $g_{0}$ in the space of Einstein metrics on $\mathrm{M}$ to a neighborhood of $h_{0}$ in the space of metrics on $\partial \mathrm{M}$.

Each example of Einstein manifold with negative curvature and convex, umbilical boundary satisfying (1) provides an application of this result. For instance, starting with a hyperbolic ball leads to:

Example 1. Let $h_{0}$ be the canonical metric on $\mathrm{S}^{m}$, and choose $\mathrm{R}>0$. There exists a neighborhood $U_{0}$ of $\mathrm{R}^{m} h_{0}$ in $\Gamma^{\infty}\left(\mathrm{S}^{2} \mathrm{~T}^{*} \mathrm{~S}^{m}\right)$ such that each $h \in U_{0}$ is induced by an Einstein metric $g$ with $\operatorname{ric}_{g}=-m g$ on $\mathrm{B}^{m+1}$.

Theorem 3 can also be used to understand Einstein deformations of "fuchsianlike" group actions on $H^{m+1}$. Namely, if a group $\Gamma$ has a discrete co-compact action $\rho$ on $H^{m}$, then $\rho$ extends naturally to an action on $H^{m+1}$ leaving invariant a totally geodesic hyperplane $\mathrm{P}_{0} \simeq H^{m}$. The set $\tilde{\mathrm{M}}_{0}$ of points of $H^{m+1}$ at distance at most $r_{0}$ of $\mathrm{P}_{0}$ has a convex, umbilical boundary, and so has the quotient $\mathrm{M}_{0} \simeq \tilde{\mathrm{M}}_{0} / \rho(\Gamma)$. The metric induced on $\partial \mathrm{M}_{0}$ has constant sectional curvature $-1+\tanh ^{2}\left(r_{0}\right)$. Now:

Example 2. There exists a neighborhood $U_{0}$ of $h_{0}$ in $\Gamma^{\infty}\left(\mathrm{S}^{2} \mathrm{~T}^{*} \partial \mathrm{M}_{0}\right)$ such that each $h \in U_{0}$ is induced by an Einstein metric $g$ with ric $_{g}=-m g$ on $\mathrm{M}_{0}$.

Other examples of Einstein metrics with umbilical boundary are provided by the following extension of the previous example (see e.g. [RS98] for a proof, it is also almost in [Bes87]):

Proposition 1. Let $h_{0}$ be an Einstein metric with ric $_{h_{0}}=-(m-1) h_{0}$ on a $m$-manifold $N$. Let $\mathrm{M}=N \times\left[-r_{0}, r_{0}\right]$ with the "warped product" metric:

$$
g_{0}=d t^{2}+\cosh ^{2}(t) h_{0} .
$$

$\left(\mathrm{M}, g_{0}\right)$ is an Einstein manifold (with ric $\left._{g_{0}}=-m g_{0}\right)$ with umbilical boundary. 
Theorem 3 can of course be used in this setting, too, if $h_{0}$ is close enough to having constant curvature, since then $g_{0}$ satisfies (1).

The result in the Ricci-flat case is slightly weaker than in the negatively curved case:

Theorem 4. Let $h_{0}$ be the canonical metric on $\mathrm{S}^{m}$, and choose $\mathrm{R}>0$. There exists a neighborhood $U_{0}$ of $\mathrm{R}^{m} h_{0}$ in $\Gamma^{\infty}\left(\mathrm{S}^{2} \mathrm{~T}^{*} \mathrm{~S}^{m}\right)$ such that each $h \in U_{0}$ is induced by a Ricci-flat metric $g$ on $\mathrm{B}^{m+1}$.

Theorem 4 works with flat metrics only because the analog of the pinching hypothesis in theorem 3, applied to a Ricci-flat metric, implies that it is flat. Flat metrics are therefore necessary to show the rigidity (with respect to infinitesimal deformations preserving the induced metric on the boundary) of metrics with an umbilical boundary. On the other hand, it is known (see [RS98]) that Ricci-flat metrics on manifolds with (convex or concave) umbilical boundaries are rigid, in the sense that they admit no 1-parameter deformation preserving the induced metric on the boundary, under rather general hypothesis (for instance if their boundary is connected). The methods used to prove those rigidity results in [RS99], [RS98], based on a "Schläfli formula" for Einstein manifolds with boundaries, are very different from those we use here (using a more classical Weitzenböck formula).

As in the case of surfaces, it seems necessary to use the Nash-Moser theorem in the proof of theorems 3 and 4, and the implicit function theorem in Banach spaces does not seem to apply. This is related to a "loss of derivative" phenomenon which should be made clear in section 2. For this reason, it is not obvious how to give results outside the $\mathrm{C}^{\infty}$ category.

It would be interesting to know whether the metrics on $\mathrm{M}$ provided by theorems 3 and 4 are actually the unique Einstein metrics (of given scalar curvature) inducing $h$ on $\partial \mathrm{M}$. It should also be pointed out that theorem 1 also applies to the sphere $\mathrm{S}^{3}$, where we have no Einstein equivalent. Theorems 1 and 2 have Lorentz analogs, too, where $H^{3}$ or $\mathbf{R}^{3}$ are replaced by one of the Lorentz spaceforms $\mathbf{R}_{1}^{3}, H_{1}^{3}$ or $\mathrm{S}_{1}^{3}$ (see [Sch96], [LS00]). Again, analogs of theorem 3 and 4 might exist in this setting, with a Lorentz metric on $\mathrm{M}$.

Another possible interpretation of the lorentzian results in dimension three, however, can be obtained through a classical duality. They indicate that an interesting phenomenon also happens (at least in some cases) when one tries to replace the metric induced on the boundary in theorem 1 by its third fundamental form. For instance, if $h$ is a smooth metric on $\mathrm{S}^{2}$ with curvature $\mathrm{K}<1$ and if all closed geodesics of $\left(\mathrm{S}^{2}, h\right)$ have length $\mathrm{L}>2 \pi$, then there exists a unique hyperbolic metric $g$ on $\mathrm{B}^{3}$ such that the third fundamental form of $\partial \mathrm{B}^{3}$ is $h$ (see [Sch96]). Moreover, a direct consequence of [LS00] is that, if $\Sigma$ is a compact surface of genus at least 2 , and if $h$ is a smooth metric on $\Sigma$ with curvature $\mathrm{K}<1$ such that all closed geodesics of $(\tilde{\Sigma}, h)$ have $\mathrm{L}>2 \pi$, then there exists a unique "fuchsian" hyperbolic metric $g$ on $\Sigma \times[-1,1]$ such that the third fundamental 
form of each connected component of the boundary is $h$; the condition on the lengths of the closed geodesics is necessary here, see [Sch96, LS00, Sch98]. Thus one might wonder whether analogs of theorems 3 and 4 are possible with the third fundamental form instead of the induced metric on the boundary.

The results above can be compared to those of Graham and Lee [GL91], who show that the conformal structure at infinity of $H^{n}$, when slightly changed, remains the conformal structure at infinity of a complete, Einstein, asymptotically hyperbolic metric. This result has recently been extended by O. Biquard [Biq00] to other rank-one symmetric spaces of non-compact type. It would be interesting to know whether theorems 3 and 4 can also be extended to deformations of e.g. Kähler-Einstein metrics.

Finally, it would be interesting to know whether the kind of results given above could be obtained by using the Ricci flow, with boundary conditions implying e.g. that the boundary remains umbilical. The relationship between the Ricci flow and Einstein manifolds is particularly clear in the work of R. Ye [Ye93], and Y. Shen [She96] proved a short time existence result for the Ricci flow on manifolds with umbilical boundary that might prove very relevant here.

This paper was significantly improved thanks to many important remarks from an anonymous referee. I would like to thank him for his efforts.

\section{Deformations of Einstein metrics}

This section contains the basic setup necessary to understand, from an analytical viewpoint, the deformations of Einstein manifolds, with scalar curvature $m(m+$ $1) \mathrm{K}_{0}$, where $\mathrm{K}_{0}=-1$, on the $(m+1)$-manifold with boundary $(\mathrm{M}, \partial \mathrm{M})$. We then give the outline of the proof of the main result, leaving the most technical parts for the next sections. We give at the end of the section some details on the modifications necessary to handle the case $\mathrm{K}_{0}=0$.

We will use an implicit function theorem, in the Nash-Moser category, which is very similar to theorem 3.3.4 in [Ham82], and we refer the reader to [Ham82] for the definitions of tame Fréchet manifolds, etc. Let $\mathcal{F}$ be a smooth tame Fréchet manifold, let $\mathcal{G}$ be a smooth tame Fréchet space, and let $\mathcal{V}$ be a smooth tame vector bundle over $\mathcal{F}$, with a smooth tame connection $\Gamma$. Let $\mathrm{P}: \mathcal{F} \rightarrow \mathcal{G}$ be a smooth tame map of manifolds, and let $Q: \mathcal{F} \rightarrow \mathcal{V}$ be a smooth tame section. Suppose that the map:

$$
\mathrm{DP} \times \mathrm{D}^{\Gamma} Q: \mathrm{T} \mathcal{F} \rightarrow \mathcal{G} \times \mathcal{V}
$$

is a smooth tame linear vector bundle morphism which is an isomorphism at points where $Q=0$, and that there exists another smooth tame linear vector bundle morphism $\mathrm{V}$ which is an approximate left and right inverse of $\mathrm{DP} \times \mathrm{D}^{\Gamma} Q$, in the 
sense that, for all $f \in \mathcal{F}$ and for all $(g, v) \in \mathcal{G} \times \mathcal{V}_{f}$ :

$$
\left(\mathrm{D}_{f} \mathrm{P} \times \mathrm{D}^{\Gamma} Q\right) \circ \mathrm{V}(g, v)=(g, v)+q^{r}(Q(f),(g, v)),
$$

and that, for all $f \in \mathcal{F}$ and all $\mathrm{F} \in \mathrm{T}_{f} \mathcal{F}$ :

$$
\mathrm{V} \circ\left(\mathrm{D}_{f} \mathrm{P} \times \mathrm{D}^{\Gamma} Q\right)(\mathrm{F})=\mathrm{F}+q^{l}(Q(f), \mathrm{F}),
$$

where $q^{l}$ and $q^{r}$ are smooth tame bilinear maps at each point $f \in \mathcal{F}$, and are smooth tame sections of the corresponding bundles over $\mathcal{F}$, i.e. $\mathcal{V}^{*} \otimes(\mathcal{G} \times \mathcal{V})^{*} \otimes$ $(\mathcal{G} \times \mathcal{V})$ and $\mathcal{V}^{*} \otimes \mathrm{T}^{*} \mathcal{F} \otimes \mathrm{T} \mathcal{F}$ respectively. Then:

Theorem 5. If $f_{0} \in \mathcal{F}$ is such that $\mathrm{P}\left(f_{0}\right)=g_{0} \in \mathcal{G}$ and that $Q\left(f_{0}\right)=0$, then there exist neighborhoods $\mathrm{W}_{f}$ and $\mathrm{W}_{g}$ of $f_{0}$ and $g_{0}$ respectively, such that, for each $g \in \mathrm{W}_{g}$, there exists a unique $f \in \mathrm{W}_{f}$ such that $Q(f)=0$ and that $\mathrm{P}(f)=g$. Moreover, the solution $f=\mathrm{S}(g)$ is defined by a smooth tame map from $\mathrm{W}_{g}$ to $\mathrm{W}_{f}$.

The proof of this theorem can be done just like the proof of theorem 3.3 .4 in [Ham82]. More precisely, theorem 3.3.4 of [Ham82] is proved by reducing it to an application of theorem 3.3.1, which contains a quadratic error; this quadratic error is necessary to prove theorem 3.3.4 because such an error comes from the choice of the connection $\Gamma$. Theorem 5 can likewise be proved as an application of theorem 3.3.1 of [Ham82], with an additional quadratic error term coming from the error term in theorem 5 .

In the proof of theorems 3 and $4, \mathcal{F}$ is the space of (smooth) Riemannian metrics on $\mathrm{M}$ (modulo diffeomorphisms fixing $\partial \mathrm{M}$ ), while $\mathcal{G}$ is the space of (smooth) metrics on $\partial \mathrm{M} . \mathcal{V}$ is the bundle over $\mathcal{F}$ of sections of the bundle of symmetric quadratic forms over $\mathrm{M}$ satisfying an equation (depending on a metric $g$ on $\mathrm{M}$ ) which is always realized for $\mathrm{ric}_{g}-m \mathrm{~K}_{0} g . \mathrm{P}$ is the map sending a metric on $\mathrm{M}$ to the induced metric on $\partial \mathrm{M}$, and $Q$ is the section of $\mathcal{V}$ sending $g$ to $\operatorname{ric}_{g}-m \mathrm{~K}_{0} g$.

We now introduce some notations that will be useful in the proof. If $g$ is a metric on $\mathrm{M}$, we call $\delta_{g}$ the divergence acting on symmetric 2 -forms on $\mathrm{M}$; if $\left(e_{1}, \cdots, e_{m+1}\right)$ is a moving frame on $\mathrm{M}$, then:

$$
\left(\delta_{g} h\right)(x)=-\sum_{i=1}^{m+1}\left(\mathrm{D}_{e_{i}} h\right)\left(x, e_{i}\right),
$$

where $\mathrm{D}$ is the Levi-Civita connection of $g$. The adjoint $\delta_{g}^{*}$ of $\delta_{g}$ acts on 1 -forms as:

$$
\left(\delta_{g}^{*} w\right)(x, y)=\frac{1}{2}\left(\left(\mathrm{D}_{x} w\right)(y)+\left(\mathrm{D}_{y} w\right)(x)\right)
$$

We also call: 
- $\mathcal{M}$ the space of compact, (smooth) Riemannian metrics on $(\mathrm{M}, \partial \mathrm{M})$ with sectional curvature $\mathrm{K}<-k_{0}$ and Ricci curvature ric $<-k_{0}$, for a constant $k_{0}>0$ small enough (e.g. $\left.k_{0}=0.1\right)$, and such that $\partial \mathrm{M}$ is strictly convex. $\mathcal{M}$ is a tame Fréchet manifold.

- $\mathcal{R}$ the space of (smooth) sections of $\mathrm{S}^{2} \mathrm{~T}^{*} \mathrm{M}$, i.e. quadratic forms on the tangent of $\mathrm{M}$, so that $\mathcal{M} \subset \mathcal{R}$.

- $\mathbf{M}_{g}=\mathrm{T}_{g} \mathcal{M}$ the tangent space to $\mathcal{M}$ at $g \in \mathcal{M}$. $\mathbf{M}_{g}$ almost does not depend on $g$, it is canonically isomorphic to $\mathcal{R}$ for all $g$, but nonetheless we consider $\mathbf{M}_{g}$ as the fiber over $g \in \mathcal{M}$ of a bundle $\mathbf{M}$ over $\mathcal{M}$.

- $\mathcal{D}_{0}$ the group of smooth diffeomorphisms of $\mathrm{M}$ fixing $\partial \mathrm{M}$.

- $\mathbf{V}_{0}=\mathrm{T}_{\mathrm{Id}} \mathcal{D}_{0}$ the space of smooth vector fields on $\mathrm{M}$ vanishing on the boundary, acting on $\mathcal{M}$ by pull-back.

- $\mathbf{V}_{\perp}$ the space of smooth vector fields on $\mathrm{M}$ orthogonal to the boundary.

- $\mathcal{M}_{0}=\mathcal{M} / \mathcal{D}_{0}$ the space of metrics modulo diffeomorphisms fixing $\partial \mathrm{M}$.

- $\mathbf{M}_{g, \delta}$ the space of elements $\mathrm{G} \in \mathbf{M}_{g}$ such that $2 \delta_{g} \mathrm{G}+d \operatorname{tr}_{g} \mathrm{G}=0$. We consider it as the fiber at $g$ of a vector bundle $\mathbf{M}_{\delta}$ over $\mathcal{M}$.

- $\mathbf{M}_{g, \delta, t}$ the space of elements $\mathrm{G} \in \mathbf{M}_{g, \delta}$ such that $\operatorname{tr}_{g} \mathrm{G}=0$ on $\partial \mathrm{M}$, also considered as the fiber at $g$ of a vector bundle $\mathbf{M}_{\delta, t}$ over $\mathcal{M}$.

- $\mathcal{R}_{g, \delta}$ the space of elements $\mathrm{R} \in \mathcal{R}$ such that $\left(2 \delta_{g}+d \operatorname{tr}_{g}\right) \mathrm{R}=0 ; \mathcal{R}_{g, \delta}$ is canonically isomorphic to $\mathbf{M}_{g, \delta}$, but we keep two notations because $\mathbf{M}_{g, \delta}$ will be considered as the tangent space to $\mathcal{M}_{0}$ at $g$ and will contain the variations of $g$, while $\mathcal{R}_{g, \delta}$ will be seen as the fibre over $g$ of a bundle $\mathcal{R}_{\delta}$ over $\mathcal{M}$ and will contain the variations of $\operatorname{ric}_{g}-m \mathrm{~K}_{0} g$.

- $\mathcal{N}$ the space of Riemannian metrics on $\partial \mathrm{M}$.

- $\mathbf{N}_{h}=\mathrm{T}_{h} \mathcal{N}$ for $h \in \mathcal{N}$.

- $\partial: \mathcal{M} \rightarrow \mathcal{N}$ the operator sending a metric on $\mathrm{M}$ to the induced metric on $\partial \mathrm{M}$, and also the linearized operator from $\mathbf{M}_{g}$ to $\mathbf{N}_{\partial g}$. When $g$ is implicit, we might use I instead of $\partial g$, since $\partial g$ is called the "first fundamental form" of the immersion of $\partial \mathrm{M}$ in $\mathrm{M}$. Using $\partial$, we obtain a bundle $\mathbf{N}$ over $\mathcal{M}$, with fiber $\mathbf{N}_{\partial g}$ at $g \in \mathcal{M}$.

- for $h \in \mathcal{N}, \mu \in \mathbf{N}_{h}, \mathbf{N}_{h, \mu}=\mathbf{N}_{h} / \mu \mathrm{C}^{\infty}(\partial \mathrm{M})$, where a function $f$ on $\partial \mathrm{M}$ acts on $\mathbf{N}_{h}$ by $n \mapsto n+f \mu$.

When needed, we will denote with an exponent $k$ the set of sections of any of the functional spaces above which are in the Sobolev space $H^{k}$ (up to the boundary).

Proposition 2. $\mathcal{M}_{0}$ is a tame Fréchet manifold. $\mathbf{M}, \mathbf{M}_{\delta}, \mathbf{M}_{\delta, t}, \mathbf{N}$ and $\mathcal{R}_{\delta}$ are smooth, tame vector bundles over $\mathcal{M}$. Moreover, they are equivariant under the action of $\mathcal{D}_{0}$, and thus define smooth, tame vector bundles over $\mathcal{M}_{0}$ which we denote by $\mathbf{M}^{0}, \mathbf{M}_{\delta}^{0}, \mathbf{M}_{\delta, t}^{0}, \mathbf{N}^{0}$ and $\mathcal{R}_{\delta}^{0}$ respectively.

The proof is in section 3 .

A basic point is that the tangent space to $\mathcal{M}_{0}$ at a point $g$ can be identified with $\mathbf{M}_{g, \delta}^{0}$. This is done as follows. 


\section{Lemma 1.}

1. Let $g_{0} \in \mathcal{M}_{0}$, and let $g \in \mathcal{M}$ an element of the equivalence class $g_{0}$. Then $\mathrm{T}_{g_{0}} \mathcal{M}_{0} \simeq \mathbf{M}_{g} / \mathbf{V}_{0}$.

2. For each $k \in \mathbf{N}^{*}$ and each $\mathrm{G} \in \mathbf{M}_{g}^{k}$, there exists a unique vector field $\mathrm{V} \in$ $\mathbf{V}_{0}^{k+1}$ such that $\mathrm{G}+2 \delta_{g}^{*} \mathrm{~V} \in \mathbf{M}_{g, \delta}^{k}$.

3. The map $\Pi_{g}: \mathrm{G} \mapsto \mathrm{G}+2 \delta_{g}^{*} \mathrm{~V}$ defines a projection from $\mathbf{M}_{g}^{k}$ to $\mathbf{M}_{g, \delta}^{k}$ which is a smooth, tame bundle morphism over $\mathcal{M}$.

4. $\Pi_{g}$ is equivariant under $\mathcal{D}_{0}$, and thus defines a map $\Pi_{g}^{0}: \mathbf{M}_{g}^{0} \rightarrow \mathbf{M}_{g, \delta}^{0}$ which is a smooth, tame bundle morphism over $\mathcal{M}_{0}$.

5. For each $k \in \mathbf{N}^{*}$ and each $g \in \mathcal{M}$, there exists $\mathrm{C}>0$ such that, for all $\mathrm{G} \in \mathbf{M}_{g}^{k}:$

$$
\left\|\mathrm{G}-\Pi_{g}(\mathrm{G})\right\|_{H^{k}} \leq \mathrm{C}\left\|\left(2 \delta_{g}+d \operatorname{tr}_{g}\right) \mathrm{G}\right\|_{H^{k-1}} .
$$

The proof of this lemma is in section 3 .

We also need a connection $\Gamma$ on $\mathcal{R}_{g, \delta}$; we can define one as follows. Let $\left(g_{t}\right)_{t \in[0,1]}$ be a smooth one-parameter family of metrics in $\mathcal{M}$, and let $\left(r_{t}\right)_{t \in[0,1]}$ be a smooth one-parameter family in $\mathcal{R}$ such that $r_{t} \in \mathcal{R}_{g_{t}, \delta} . \quad r_{0}^{\prime} \in \mathcal{R}_{g} \simeq \mathbf{M}_{g}$, so we define $\mathrm{D}_{g_{0}^{\prime}}^{\Gamma} r:=\Pi_{g}\left(r_{0}^{\prime}\right)$. Then $\mathrm{D}_{g_{0}^{\prime}}^{\Gamma} r \in \mathbf{M}_{g_{0}, \delta} \equiv \mathcal{R}_{g_{0}, \delta}$.

Proposition 3. $\Gamma$ defines a smooth and tame connection on $\mathcal{R}_{\delta}$ as a bundle over $\mathcal{M}$, and also on $\mathcal{R}_{\delta}^{0}$ as a bundle over $\mathcal{M}_{0}$. For each $g \in \mathcal{M}$ and each $k \in \mathbf{N}^{*}$, there exists $\mathrm{C}>0$ such that, in the setting described above:

$$
\left\|r_{0}^{\prime}-\mathrm{D}_{g_{0}^{\prime}}^{\Gamma} r\right\|_{H^{k}} \leq \mathrm{C}\left\|\left(2 \delta_{g_{0}}+d \operatorname{tr}_{g_{0}}\right) r_{0}^{\prime}\right\|_{H^{k-1}} .
$$

Its definition shows that $\Gamma$ defines a smooth, tame connection on $\mathcal{R}_{\delta}$, while point (4) of lemma 1 indicates that it has the required equivariance property to define a (smooth, tame) connection on $\mathcal{R}_{\delta}^{0}$. The upper bound comes from point (5) of lemma 1.

For $g \in \mathcal{M}$, define $r(g):=\operatorname{ric}_{g}-m \mathrm{~K}_{0} g . r$ will be the section $Q$ of $\mathcal{V}$ used in theorem 5. Taking the trace of the differential Bianchi identity shows that:

$$
\left(2 \delta_{g}+d \operatorname{tr}_{g}\right) \text { ric }_{g}=0
$$

while it is clear that:

$$
\left(2 \delta_{g}+d \operatorname{tr}_{g}\right) g=0 .
$$

Therefore $r$ is a section of $\mathcal{R}_{g, \delta}$ as a vector bundle over $\mathcal{M}$. Moreover, it is invariant under the action of $\mathcal{D}_{0}$, because, for $u \in \mathcal{D}_{0}$ and $g \in \mathcal{M}$ :

$$
u^{*} \operatorname{ric}_{g}=\operatorname{ric}_{u^{*} g} \text {. }
$$

This shows that $r$ is a section of $\mathcal{R}_{\delta}^{0}$. It is then easy to show from its definition that: 
Proposition 4. $r$ is a smooth, tame section of $\mathcal{R}_{\delta}^{0}$.

There is a natural operator $\partial: \mathcal{M} \rightarrow \mathcal{N}$ sending a metric $g \in \mathcal{M}$ to the induced metric $\partial g$ on $\partial \mathrm{M}$. Since $\mathcal{D}_{0}$ is made of diffeomorphisms fixing the boundary, $\partial$ is invariant under the action of $\mathcal{D}_{0}$ on $\mathcal{M}$, so $\partial$ can be considered as an operator from $\mathcal{M}_{0}$ to $\mathcal{N}$. We will also call $\partial$ the linearized operator $\mathrm{T}_{g} \mathcal{M}_{0} \rightarrow \mathrm{T}_{\partial g} \mathcal{N}$ at a point $g \in \mathcal{M}_{0}$. $\partial$ will be the operator $\mathrm{P}$ appearing in theorem 5 . We leave to the reader the proof of the:

Proposition 5. $\partial$ is a smooth, tame operator from $\mathcal{M}_{0}$ to $\mathcal{N}$.

From now on, we call II the second fundamental form of the boundary $\partial \mathrm{M}$. When $g \in \mathcal{M}_{0}$ is such that $\operatorname{ric}_{g} \leq 0$ and that $\partial \mathrm{M}$ is strictly convex for $g$, finding $\mathrm{G} \in \mathbf{M}_{g, \delta}$ such that $\partial \mathrm{G}=H$ (for a given $H \in \mathbf{N}_{\partial g}$ ) boils down to finding $\mathrm{G}^{\prime} \in \mathbf{M}_{g, \delta}$ such that $\partial \mathrm{G}^{\prime} \equiv H \bmod \mathbb{I}$, i.e. such that there exists $f \in \mathrm{C}^{\infty}(\partial \mathrm{M})$ with $\partial \mathrm{G}^{\prime}=H+f \mathbb{I}$. This is because of the:

Lemma 2. Suppose that $g \in \mathcal{M}$ has $\operatorname{ric}_{g} \leq 0$, and that $\partial \mathrm{M}$ is strictly convex for $g$. Let $\mathrm{G}^{\prime} \in \mathbf{M}_{g, \delta}$. There is a unique $\mathrm{V} \in \mathbf{V}_{\perp}$ such that $\mathrm{G}:=\mathrm{G}^{\prime}+2 \delta_{g}^{*} \mathrm{~V} \in$ $\mathbf{M}_{g, \delta, t} ; \mathrm{V}=f n$ on $\partial \mathrm{M}$ for some $f \in \mathrm{C}^{\infty}(\partial \mathrm{M})$. Moreover, $\partial \mathrm{G}=\partial \mathrm{G}^{\prime}-2 f \mathrm{II}$. The mapping:

$$
\begin{aligned}
\mathcal{F}_{g}: \mathbf{M}_{g, \delta} & \rightarrow \mathbf{M}_{g, \delta, t} \times \mathrm{C}^{\infty}(\partial \mathrm{M}) \\
\mathrm{G}^{\prime} & \mapsto(\mathrm{G}, f)
\end{aligned}
$$

defines a smooth, tame isomorphism of vector bundles over $\mathcal{M}$. Moreover, it is equivariant under $\mathcal{D}_{0}$ and thus defines a smooth, tame vector bundle isomorphism $\mathcal{F}^{0}: \mathbf{M}_{\delta}^{0} \rightarrow \mathbf{M}_{\delta, t}^{0} \times \mathrm{C}^{\infty}(\partial \mathrm{M})$.

The proof is also in section 3. Note that using metric variations $G$ with $\operatorname{tr}_{g}(\mathrm{G})=0$ on $\partial \mathrm{M}$ makes sense because, when the variation $\mathrm{G}$ of $g$ is subject to the equations implying that $g$ remains Einstein, its trace on $M$ is essentially determined by its trace on $\partial \mathrm{M}$. This point should become clear at the beginning of section 5. It will not, however, be used formally in the proof.

We now have a section $r$ of the vector bundle $\mathcal{R}_{\delta}^{0}$ over $\mathcal{M}_{0}$, and an operator $\partial: \mathcal{M}_{0} \rightarrow \mathcal{N}$. Let $g \in \mathcal{M}$. Let $h=\partial g \in \mathcal{N}$. We will now define a linear operator:

$$
\mathrm{V}_{g}: \mathrm{T}_{\partial g} \mathcal{N} \times \mathcal{R}_{g, \delta} \rightarrow \mathbf{M}_{g, \delta}
$$

and show later that $\mathrm{V}_{g}$ defines a bundle morphism $\mathrm{V}_{g}^{0}: \mathrm{TN}^{0} \times \mathcal{R}_{\delta}^{0} \rightarrow \mathbf{M}_{\delta}^{0}$ which is an approximate left and right inverse of $\partial \times \mathrm{D}^{\Gamma} r$ (with a quadratic error term as in theorem 5).

The infinitesimal variation of $r$ associated to an infinitesimal variation $G$ of 
$g$ is given by the following well-known formula (see [Bes87]):

$$
r^{\prime}=\mathrm{D}_{g}^{*} \mathrm{D}_{g} \mathrm{G}-2 \stackrel{\circ}{\mathrm{R}} g \mathrm{G}-2 \delta_{g}^{*} \delta_{g} \mathrm{G}-\mathrm{D}_{g} d \operatorname{tr}_{g}(\mathrm{G}) .
$$

A classical problem here is that this operator is elliptic in $G$ but strongly degenerate, because of its invariance under the group of diffeomorphisms of $M$. The "classical" solution (see [DeT81, GL91, Bes87, Biq00]) is to introduce another, elliptic non-degenerate problem, and to show that its solutions are actually solutions of the original problem. This should be done here with some care regarding the boundary conditions. Namely, one should add some conditions at the boundary which will later ensure that, when $g$ is Einstein, solutions of the "new" (elliptic non-degenerate) system are also solutions of the elliptic degenerate system - this is basically the content of proposition 6 below, although this proposition is not used formally in the proof.

Moreover, the equation which is then obtained is elliptic non-degenerate in $\mathrm{M}$, but is still not an elliptic boundary problem. To get one, it is necessary to "get rid of" the trace part of the metric variation, so as to obtain on the boundary a metric variation which is the desired one, but only on the orthogonal to the second fundamental form. It is then necessary to add a normal deformation of $\partial \mathrm{M}$ in $\mathrm{M}$ to obtain the full variation of the metric induced on the boundary.

Let $g \in \mathcal{M}$, and let $(H, \mathrm{R}) \in \mathrm{T}_{\partial g} \mathcal{N} \times \mathcal{R}_{g, \delta}$. Consider the following system:

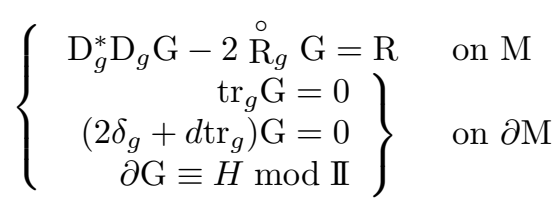

Then:

Lemma 3. (6) is an elliptic boundary problem on $\mathrm{M}$ with index 0 .

The proof is in section 4 .

Lemma 4. If $r(g)=0$ and $\partial \mathrm{M}$ is strictly convex and umbilical for $g$, and if $g$ satisfies (1) (or (2)), then the only solution of (6) for $\mathrm{R} \equiv 0$ and $H \equiv 0$ is $\mathrm{G} \equiv 0$.

The proof is in section 5. As a consequence, we have by the open mapping theorem that:

Corollary 1. There is a neighborhood $\mathrm{W}_{g}$ of $g$ in $\mathcal{M}$ such that, for $g^{\prime} \in \mathrm{W}_{g}$, the only solution of (6) for $\mathrm{R} \equiv 0$ and $H \equiv 0$ is $\mathrm{G} \equiv 0$.

All this shows that, for $g^{\prime} \in \mathrm{W}_{g}$ and $(H, \mathrm{R}) \in \mathbf{N}_{\partial g^{\prime}} \times \mathcal{R}_{g^{\prime}, \delta}$, there is a unique solution $\mathrm{G}^{\prime} \in \mathbf{M}_{g}$ of $(6)$ and a unique $f \in \mathrm{C}^{\infty}(\partial \mathrm{M})$ such that $H=\partial \mathrm{G}^{\prime}-2 f I$. 
Let $\mathrm{V}_{g^{\prime}}(H, \mathrm{R}):=\Pi_{g^{\prime}}\left(\mathrm{G}^{\prime}\right)+\mathcal{F}_{g^{\prime}}^{-1}(0, f)$ (where $\mathcal{F}_{g^{\prime}}$ comes from lemma 2). This defines a mapping $\mathrm{V}_{g}: \mathbf{N}_{\partial g^{\prime}} \times \mathcal{R}_{g^{\prime}, \delta} \rightarrow \mathbf{M}_{g^{\prime}, \delta}$.

Moreover, it is not difficult to check that equation (6), being defined geometrically, is equivariant under the action of $\mathcal{D}_{0}$. Together with point (4) of lemma 1 , this show that the definition of $\mathrm{V}$ is also equivariant. Therefore, it actually defines a bundle morphism over $\mathcal{M}_{0}$. Thus:

Lemma 5. $\mathrm{V}_{g^{\prime}}$ is smooth and tame. The bundle morphism $\mathrm{V}$ is equivariant under $\mathcal{D}_{0}$ and thus defines a smooth, tame bundle morphism $\mathrm{V}^{0}: \mathbf{N}^{0} \times \mathcal{R}_{\delta}^{0} \rightarrow \mathbf{M}_{\delta}^{0}$.

Finally, we will check in section 6 the following lemma:

Lemma 6. Let $g_{0} \in \mathcal{M}_{0}$. There exists a neighborhood $\mathrm{W}_{g_{0}}$ of $g_{0}$ in $\mathcal{M}_{0}$ such that, for each $g \in \mathrm{W}_{g_{0}}$, there exist smooth, tame bilinear maps:

$$
q_{g}^{l}: \mathcal{R}_{g, \delta}^{0} \times \mathbf{M}_{g, \delta}^{0} \rightarrow \mathbf{M}_{g, \delta}^{0}
$$

and:

$$
q_{g}^{r}: \mathcal{R}_{g, \delta}^{0} \times\left(\mathcal{N}_{\partial g} \times \mathcal{R}_{g, \delta}^{0}\right) \rightarrow\left(\mathcal{N}_{\partial g} \times \mathcal{R}_{g, \delta}^{0}\right)
$$

such that $q^{r}$ and $q^{l}$ are smooth, tame sections of the corresponding vector bundles over $\mathcal{M}_{0}$ and that, for each $\mathrm{G} \in \mathbf{M}_{g, \delta}^{0}$ :

$$
\mathrm{V}_{g}^{0} \circ\left(\partial \times \mathrm{D}^{\Gamma} r\right) \mathrm{G}=\mathrm{G}+q_{g}^{l}(r(g), \mathrm{G})
$$

and, for each $(H, \mathrm{R}) \in \mathcal{N}_{\partial g} \times \mathcal{R}_{g, \delta}^{0}$ :

$$
\left(\partial \times \mathrm{D}^{\Gamma} r\right) \circ \mathrm{V}_{g}^{0}(H, \mathrm{R})=(H, \mathrm{R})+q_{g}^{r}(r(g),(H, \mathrm{R}))
$$

An important motivation for the proof of this lemma is the following remarkable fact, which appeared in slightly different forms in previous works (see e.g. [Bes87, Biq00]). It implies for instance that, when $r=0$, a solution of $\mathrm{G}$ (6) has $(2 \delta+d \operatorname{tr}) \mathrm{G}=0$ not only on $\partial \mathrm{M}$, but in the whole of $\mathrm{M}$, so that $\mathrm{R}=r^{\prime}$. The proof is in section 6. It is not, however, used formally in the proof.

Proposition 6. Let $g \in \mathcal{M}$ and $\mathrm{G} \in \mathbf{M}_{g}$. Suppose that:

$$
\mathrm{D}_{g}^{*} \mathrm{D}_{g} \mathrm{G}-2 \stackrel{\circ}{\mathrm{R}} \mathrm{G}=\mathrm{R}
$$

with:

$$
\left(2 \delta_{g}+d \operatorname{tr}_{g}\right) \mathrm{R}+\left(2 \delta_{g}^{\prime}+d \operatorname{tr}_{g}^{\prime}\right) r(g)=0,
$$

where $\delta_{g}^{\prime}$ and $\operatorname{tr}_{g}^{\prime}$ are the variations of $\delta_{g}$ and $\operatorname{tr}_{g}$ corresponding to the variation $\mathrm{G}$ of $g$. Suppose further that:

$$
\left(2 \delta_{g}+d \operatorname{tr}_{g}\right) \mathrm{G}=0 \text { on } \partial \mathrm{M} .
$$

Then $\left(2 \delta_{g}+d \operatorname{tr}_{g}\right) \mathrm{G}=0$ on $\mathrm{M}$, so that $\mathrm{G} \in \mathbf{M}_{g, \delta}$. 
We can now apply the Nash-Moser implicit function theorem 5 , with $\mathrm{P}=\partial$ and $Q=r$; propositions 4 and 5 show that $\partial \times \mathrm{D}^{\Gamma} r$ is a smooth tame bundle morphism, while $\mathrm{V}_{g}$ is the approximate local inverse we need. Note that lemma 6 implies that, when $r=0, \mathrm{~V}_{g}^{0}$ is actually the inverse of $\partial \times \mathrm{D}^{\Gamma} r$, which is then an isomorphism. This leads to theorem 3 and 4 , which we can reformulate in the following slightly more precise way:

Theorem 6. Let $g_{0} \in r^{-1}(0) \subset \mathcal{M}_{0}$ be such that $\partial \mathrm{M}$ is (strictly) convex and umbilical. Suppose that either $\mathrm{K}_{0}=-1$ and $g$ satisfies (1) (or (2)), or $\mathrm{K}_{0}=0$ and $g$ is flat. Then $\partial$ is a bijection from a neighborhood of $g_{0}$ in $r^{-1}(0)$ to a neighborhood of $\partial g_{0}$ in $\mathcal{N}$.

It should be pointed out that the method described above, although it might seem complicated, is necessary because a straightforward approach based on the implicit function theorem for Banach spaces (directly solving an elliptic problem with some additional condition on the boundary so that $\mathrm{G}$ induces a given metric variation on $\partial \mathrm{M}$ ) apparently doesn't work. This is because the degree of smoothness that one can obtain in the metric variation on $\partial \mathrm{M}$ is different for the parts of $\mathrm{G}$ corresponding respectively to the deformation of the metric in $\mathrm{M}$ (among those with a given a given trace, which vanishes on the boundary) and to the normal displacement of $\partial \mathrm{M}$. It is therefore necessary to go to the Fréchet category and use the Nash-Moser theorem. Although it might not seem too obvious, this is actually very similar to the classical approach of isometric immersions of surfaces (see [Ham82], III.2.1), where the two components that appear are the normal displacement of the surface (again) and the action of tangent vector fields, see [Ham82].

The proof in the case where $K_{0}=0$ is similar, but some modifications are necessary. The definition of $\mathcal{M}$ has to be changed, so that $\mathcal{M}$ contains a neighborhood of the metric on the unit ball in $\mathbf{R}^{n+1}$. Lemmas 1 and 2 still hold in the neighborhood of this flat metric, although the hypothesis made in the statement of lemma 2 exclude this case; more precisions are given on this point in section 3 . The lemmas 3 and 4 still apply around this flat metric, and the rest of the proof does not vary from the negatively curved case.

What remains of this paper contains the proofs of the statements above. Section 3 deals with lemmas 1 and 2 , section 4 with lemma 3 , and section 5 with lemma 4. Section 6 gives the proof of proposition 6 and of lemma 6 .

\section{Equations on vector fields}

We give in this section the proofs of lemmas 1 and 2, which involve the resolution of vector-valued elliptic equations on $\mathrm{M}$. We will be concerned first with the case $\mathrm{K}_{0}=-1$, and give at the end of the section details on what has to be changed to 
handle the case $\mathrm{K}_{0}=0$.

The first point to tackle is the proof of proposition 2 , which we recall here.

Proposition 2. $\mathcal{M}_{0}$ is a tame Fréchet manifold. $\mathbf{M}, \mathbf{M}_{g, \delta}, \mathbf{M}_{g, \delta, t}$ and $\mathcal{R}_{g, \delta}$ are equivariant under the action of $\mathcal{D}_{0}$, and thus define smooth, tame vector bundles over $\mathcal{M}_{0}$ which we denote by $\mathbf{M}^{0}, \mathbf{M}_{g, \delta}^{0}, \mathbf{M}_{g, \delta, t}^{0}$ and $\mathcal{R}_{g, \delta}^{0}$ respectively.

Remember that, if $g_{0}, g \in \mathcal{M}_{0}$, there exists a unique map $h: \mathrm{M} \rightarrow \mathrm{M}$, in the homotopy class of the identity, which is the identity on $\partial \mathrm{M}$ and which is harmonic between $\left(\mathrm{M}, g_{0}\right)$ and $(\mathrm{M}, g)$.

The existence of $h$ was proved in [Ham75], while the uniqueness comes from standard methods for harmonic maps, i.e. a negative upper bound on the Laplacian of the distance between $g_{0}$ and $g$. Note that both the existence and the uniqueness of $h$ use the curvature and convexity hypothesis appearing in the definition of $\mathcal{M}_{0}$.

Moreover, for $g$ close enough to $g_{0}, h$ is a diffeomorphism. This identifies a neighborhood of $g_{0}$ in $\mathcal{M}_{0}$ with a neighborhood of $g_{0}$ in the set $\mathcal{M}_{1}$ of metrics $g$ on $\mathrm{M}$ satisfying a partial differential operator expressing that the identity is harmonic between $g_{0}$ and $g$ (see [GL91]). Thus one can show that $\mathcal{M}_{1}$ is a tame Fréchet manifold. Finally, the manifold structures induced on $\mathcal{M}_{0}$ by different choices of $g_{0}$ can be seen to be compatible, so that $\mathcal{M}_{0}$ is a tame Fréchet manifold.

That $\mathbf{M}$ defines a smooth, tame vector bundle $\mathbf{M}^{0}$ over $\mathcal{M}_{0}$ is clear. $\mathbf{M}_{g, \delta}$ is defined by a simple first order P.D.E., it is therefore the fiber over $g \in \mathcal{M}$ of a smooth tame vector bundle $\mathbf{M}_{\delta}$ over $\mathcal{M}$. Moreover, $\mathcal{D}_{0}$ acts in a natural way on $\mathbf{M}_{\delta}$ : if $\mathrm{G} \in \mathbf{M}_{g, \delta}$ and $u \in \mathcal{D}_{0}$, then $u^{*} \mathrm{G} \in \mathbf{M}_{u^{*} g, \delta}$, because:

$$
\left(2 \delta_{u^{*} g}+d \operatorname{tr}_{u^{*} g}\right) u^{*} \mathrm{G}=u^{*}\left(\left(2 \delta_{g}+d \operatorname{tr}_{g}\right) \mathrm{G}\right)=0 .
$$

Therefore, $\mathbf{M}_{g, \delta}$ can be considered also as the fibre over $g \in \mathcal{M}_{0}$ of a bundle $\mathbf{M}_{\delta}$ over $\mathcal{M}_{0}$, and it is easy to see that $\mathbf{M}_{\delta}$ is smooth and tame.

It is also clear that $\mathbf{N}$ defines a smooth, tame bundle over $\mathcal{M}$, and quite obvious that one can associate to it a smooth, tame bundle $\mathbf{N}^{0}$ over $\mathcal{M}_{0}$, because, for $g \in \mathcal{M}$, the $\mathbf{N}_{u^{*} g}$ for different values of $u \in \mathcal{D}_{0}$ are canonically identified. Finally, the proof concerning $\mathcal{R}_{\delta}$ is almost the same as for $\mathbf{M}_{\delta}$.

Now the first result related to equations on vector fields was lemma 1.

\section{Lemma 1.}

1. Let $g_{0} \in \mathcal{M}_{0}$, and let $g \in \mathcal{M}$ an element of the equivalence class $g_{0}$. Then $\mathrm{T}_{g_{0}} \mathcal{M}_{0} \simeq \mathbf{M}_{g} / \mathbf{V}_{0}$.

2. For each $k \in \mathbf{N}^{*}$ and each $\mathrm{G} \in \mathbf{M}_{g}^{k}$ there exists a unique vector field $\mathrm{V} \in$ $\mathbf{V}_{0}^{k+1}$ such that $\mathrm{G}+2 \delta_{g}^{*} \mathrm{~V} \in \mathbf{M}_{g, \delta}^{k}$.

3. The map $\Pi_{g}: \mathrm{G} \mapsto \mathrm{G}+2 \delta_{g}^{*} \mathrm{~V}$ defines a projection from $\mathbf{M}_{g}^{k}$ to $\mathbf{M}_{g, \delta}^{k}$ which is a smooth, tame bundle morphism over $\mathcal{M}$. 
4. $\Pi_{g}$ is equivariant under $\mathcal{D}_{0}$, and thus defines a map $\Pi_{g}^{0}: \mathbf{M}_{g}^{0} \rightarrow \mathbf{M}_{g, \delta}^{0}$ which is a smooth, tame bundle morphism over $\mathcal{M}_{0}$.

5. For each $k \in \mathbf{N}^{*}$ and each $g \in \mathcal{M}$, there exists $\mathrm{C}>0$ such that, for all $\mathrm{G} \in \mathbf{M}_{g}^{k}:$

$$
\left\|\mathrm{G}-\Pi_{g}(\mathrm{G})\right\|_{H^{k}} \leq \mathrm{C}\left\|\left(2 \delta_{g}+d \operatorname{tr}_{g}\right) \mathrm{G}\right\|_{H^{k-1}} .
$$

The first point is clear, because $\mathrm{T}_{\mathrm{Id}} \mathcal{D}_{0}=\mathbf{V}_{0}$.

For the second point, we have to show that, for any $\mathbf{G} \in \mathbf{M}_{g}$, there exists a unique $\mathrm{V} \in \mathbf{V}_{0}$ such that:

$$
\left(2 \delta_{g}+d \operatorname{tr}_{g}\right)\left(\mathrm{G}+2 \delta_{g}^{*} \mathrm{~V}\right)=0,
$$

or that:

$$
2\left(2 \delta_{g} \delta_{g}^{*}-d \delta_{g}\right) \mathrm{V}=-\left(2 \delta_{g} \mathrm{G}+d \operatorname{tr}_{g} \mathrm{G}\right) .
$$

An easy computations (see e.g. [Bes87], [RS98]) shows that

$$
2 \delta_{g} \delta_{g}^{*}-d \delta_{g}=\mathrm{D}_{g}^{*} \mathrm{D}_{g}-\operatorname{ric}_{g}=\mathrm{D}_{g}^{*} \mathrm{D}_{g}-m \mathrm{~K}_{0} g,
$$

so our problem boils down to proving that there exists a unique solution of:

$$
\left\{\begin{aligned}
2\left(\mathrm{D}_{g}^{*} \mathrm{D}_{g} \mathrm{~V}-m \mathrm{~K}_{0} \mathrm{~V}\right) & =-\left(2 \delta_{g}+d \mathrm{tr}_{g}\right) \mathrm{G} & \text { on } \mathrm{M} \\
\mathrm{V} & =0 & \text { on } \partial \mathrm{M} .
\end{aligned}\right.
$$

Define:

$$
\begin{aligned}
\mathrm{F}: \quad \mathbf{V}_{0}^{1} & \rightarrow \mathbf{R} \\
\mathrm{V} & \mapsto \int_{\mathrm{M}}\left\|\mathrm{D}_{g} \mathrm{~V}\right\|^{2}-m \mathrm{~K}_{0}\|\mathrm{~V}\|^{2} d v+\int_{\mathrm{M}}\left\langle\left(2 \delta_{g}+d \mathrm{tr}_{g}\right) \mathrm{G}, \mathrm{V}\right\rangle d v .
\end{aligned}
$$

$\mathrm{F}$ is strictly convex, and moreover it is coercive; this is clear if $\mathrm{K}_{0}<0$, and, if $\mathrm{K}_{0}=0$, it follows from the Poincaré inequality for vector fields vanishing on $\partial \mathrm{M}$ :

$$
\exists \mathrm{C}, \forall v \in \mathbf{V}_{0}^{1}, \int_{\mathrm{M}}\langle\mathrm{D} v, \mathrm{D} v\rangle \geq \mathrm{C} \int_{\mathrm{M}}\langle v, v\rangle d \mathrm{~V} .
$$

Therefore, $\mathrm{F}$ admits a unique minimum $\mathrm{V}_{0}$ on $\mathbf{V}_{0}^{1}$, which for classical elliptic reasons is smooth. Then, for all $u \in \mathbf{V}_{0},\left(\mathrm{~T}_{\mathrm{V}_{0}} \mathrm{~F}\right)(u)=0$, and it follows in a very classical way that:

$$
2\left(\mathrm{D}_{g}^{*} \mathrm{D}_{g} \mathrm{~V}_{0}-m \mathrm{~K}_{0} \mathrm{~V}_{0}\right)=-2 \delta_{g} \mathrm{G}-d \operatorname{tr}_{g}(\mathrm{G})
$$

as needed.

The third point is easy: $\Pi_{g}$ is a projection because it is clearly the identity on $\mathbf{M}_{g, \delta}$, while its definition shows that it is smooth and tame.

For the fourth point, let $g \in \mathcal{M}$ and $\mathrm{G} \in \mathbf{M}_{g}$. By definition, $\Pi_{g} \mathrm{G}=$ $\mathrm{G}+2 \delta_{g}^{*} \mathrm{~V}$, where $\mathrm{V}$ is the unique element of $\mathbf{V}_{0}$ such that $\left(2 \delta_{g}+d \operatorname{tr}_{g}\right)\left(\Pi_{g} \mathrm{G}\right)=0$. Let $u \in \mathcal{D}_{0}$. Then it is straightforward to check that, because all terms are defined geometrically:

$$
\left(2 \delta_{u^{*} g}+d \operatorname{tr}_{u^{*} g}\right)\left(u^{*} \mathrm{G}+2 \delta_{u^{*} g}^{*}\left(u^{*} \mathrm{~V}\right)\right)=u^{*}\left(\left(2 \delta_{g}+d \operatorname{tr}_{g}\right)\left(\mathrm{G}+2 \delta_{g}^{*} \mathrm{~V}\right)\right)=0,
$$


so that, by definition of $\Pi$ :

$$
\Pi_{u^{*} g}\left(u^{*} \mathrm{G}\right)=u^{*}\left(\Pi_{g} \mathrm{G}\right)
$$

which is the required equivariance property.

Finally, to prove the upper bound in the fifth point, note that, by definition:

$$
\Pi_{g}(\mathrm{G})-\mathrm{G}=2 \delta_{g}^{*} \mathrm{~V},
$$

where $\mathrm{V} \in \mathbf{V}_{0}$ is such that:

$$
\left(2 \delta_{g}+d \operatorname{tr}_{g}\right)\left(2 \delta_{g}^{*} \mathrm{~V}\right)=-\left(2 \delta_{g}+d \operatorname{tr}_{g}\right) \mathrm{G}
$$

so that, by (10):

$$
2\left(\mathrm{D}_{g}^{*} \mathrm{D}_{g}-\operatorname{ric}_{g}\right) \mathrm{V}=-\left(2 \delta_{g}+d \operatorname{tr}_{g}\right) \mathrm{G} .
$$

Now $\mathrm{D}_{g}^{*} \mathrm{D}_{g}-$ ric $_{g}$ is elliptic and positive definite, so standard arguments indicate that there exists $\mathrm{C}>0$ such that $\|\mathrm{V}\|_{H^{k}} \leq \mathrm{C}\left\|\left(2 \delta_{g}+d \operatorname{tr}_{g}\right) \mathrm{G}\right\|_{H^{k-1}}$.

Finally, we need to prove the:

Lemma 2. Suppose that $g \in \mathcal{M}$ has ric $_{g} \leq 0$, and that $\partial \mathrm{M}$ is strictly convex for $g$. Let $\mathrm{G}^{\prime} \in \mathbf{M}_{g, \delta}$. There is a unique $\mathrm{V} \in \mathbf{V}_{\perp}$ such that $\mathrm{G}:=\mathrm{G}^{\prime}+2 \delta_{g}^{*} \mathrm{~V} \in$ $\mathbf{M}_{g, \delta, t} ; \mathrm{V}=f n$ on $\partial \mathrm{M}$ for some $f \in \mathrm{C}^{\infty}(\partial \mathrm{M})$. Moreover, $\partial \mathrm{G}=\partial \mathrm{G}^{\prime}-2 f \mathrm{II}$. The mapping:

$$
\begin{aligned}
\mathcal{F}_{g}: \mathbf{M}_{g, \delta} & \rightarrow \mathbf{M}_{g, \delta, t} \times \mathrm{C}^{\infty}(\partial \mathrm{M}) \\
\mathrm{G}^{\prime} & \mapsto(\mathrm{G}, f)
\end{aligned}
$$

defines a smooth, tame isomorphism of vector bundles over $\mathcal{M}$. Moreover, it is equivariant under $\mathcal{D}_{0}$ and thus defines a smooth, tame vector bundle isomorphism $\mathcal{F}^{0}: \mathbf{M}_{\delta}^{0} \rightarrow \mathbf{M}_{\delta, t}^{0} \times \mathrm{C}^{\infty}(\partial \mathrm{M})$

Since $\operatorname{tr}_{g} \delta_{g}^{*} \mathrm{~V}=-\delta_{g} \mathrm{~V}$ and $\left(2 \delta_{g}+d \operatorname{tr}_{g}\right) \delta_{g}^{*}=\mathrm{D}_{g}^{*} \mathrm{D}_{g}-\operatorname{ric}_{g}$, we only need to find $\mathrm{V} \in \mathbf{V}_{\perp}$ such that:

$$
\left\{\begin{array}{rlrl}
\mathrm{D}_{g}^{*} \mathrm{D}_{g} \mathrm{~V}-\mathrm{ric}_{g} \mathrm{~V} & =0 & & \text { on } \mathrm{M} \\
2 \delta_{g} \mathrm{~V} & =\operatorname{tr}_{g} \mathrm{G}^{\prime} & \text { on } \partial \mathrm{M} .
\end{array}\right.
$$

We will show that (11) is elliptic with index 0 , and then that solutions are unique. The existence of a solution $\mathrm{V}$ for any $\mathrm{G}^{\prime}$ follows.

Consider the auxiliary problem:

$$
\left\{\begin{array}{rlr}
\mathrm{D}_{g}^{*} \mathrm{D}_{g} \mathrm{~V}-(1-t) \text { ric }_{g} \mathrm{~V}+t \mathrm{~V} & =t \mathrm{~W} & \text { on } \mathrm{M} \\
2(1-t) \delta_{g} \mathrm{~V}-2 t\left\langle\mathrm{D}_{n} \mathrm{~V}, n\right\rangle & =(1-t) \operatorname{tr}_{g} \mathrm{G} & \text { on } \partial \mathrm{M}
\end{array}\right.
$$

for $t \in[0,1]$. (12) is an elliptic boundary value problem. We check this using the notations in [Sch95]. The principal symbol of the equation in $\mathrm{M}$ is:

$$
\operatorname{Psym}\left(\mathrm{D}^{*} \mathrm{D}\right)(x, v) \mathrm{V}=\|v\|^{2} \mathrm{~V} .
$$

Choose $x \in \partial \mathrm{M}$, and a chart around $x$ such that $\partial \mathrm{M} \simeq \mathbf{R}^{m} \subset \mathbf{R}^{m+1}$ around $x$. The space of bounded solutions of the relevant ODE at $(x, \tilde{v}) \in \mathrm{T}_{x} \partial \mathrm{M}$ is

$$
\mathcal{M}_{x, \tilde{v}}^{+}=\left\{\mathrm{V}(s) \mid \mathrm{V}_{i}(s)=\mathrm{V}_{i}^{0} e^{-\|\tilde{v}\| s}\right\}
$$


where $\mathrm{V}_{i}$ is the $i^{\text {th }}$ coordinate of $\mathrm{V}$ in the chart.

The principal symbols of the boundary conditions are $\mathrm{V}_{i}$ for $1 \leq i \leq m$ (this corresponds to the condition that $\mathrm{V} \in \mathbf{V}_{\perp}$ ), and

$$
-2 \partial_{s} \mathrm{~V}_{m+1}+2 i(1-t) \sum_{i=1}^{m} \tilde{v}_{i} \mathrm{~V}_{i} .
$$

Now the corresponding linear operator is obviously injective, so (12) is elliptic for $t \in[0,1]$.

For $t=1$ it is simply (including the condition that $\mathrm{V} \in \mathbf{V}_{\perp}$, which translates as "V $\| n$ on $\partial \mathrm{M}$ "):

$$
\left\{\begin{array}{cr}
\mathrm{D}_{g}^{*} \mathrm{D}_{g} \mathrm{~V}+\mathrm{V}=\mathrm{W} \\
-2\left\langle\mathrm{D}_{n} \mathrm{~V}, n\right\rangle=0 \\
\mathrm{~V} \| n
\end{array}\right\} \quad \begin{array}{r}
\text { on } \mathrm{M} \\
\text { on } \partial \mathrm{M} .
\end{array}
$$

It is easy to show, by the same minimization procedure as in the proof of proposition 1, that (13) has a unique solution for any W . (13) therefore has index 0 , and, by the deformation invariance of the index, (11) has index 0 too.

To prove that the solutions of (11) are unique, we have to show that any solution for $\mathrm{G}=0$ is zero. If $\mathrm{V}$ is such a solution, then:

$$
0=\int_{\mathrm{M}}\left\langle\mathrm{D}_{g}^{*} \mathrm{D}_{g} \mathrm{~V}-\operatorname{ric}_{g} \mathrm{~V}, \mathrm{~V}\right\rangle d v=\int_{\mathrm{M}}\left\|\mathrm{D}_{g} \mathrm{~V}\right\|^{2}-\left\langle\operatorname{ric}_{g} \mathrm{~V}, \mathrm{~V}\right\rangle d v+\int_{\partial \mathrm{M}}\left\langle\mathrm{D}_{n} \mathrm{~V}, \mathrm{~V}\right\rangle d a .
$$

Now $\operatorname{ric}_{g} \leq 0$, and, since $\delta_{g} \mathrm{~V}=0$, we have for any moving frame $\left(e_{i}\right)_{i \in \mathbf{N}_{m}}$ on $\partial \mathrm{M}$ :

$$
\left\langle\mathrm{D}_{n} \mathrm{~V}, \mathrm{~V}\right\rangle=f\left\langle\mathrm{D}_{n} \mathrm{~V}, n\right\rangle=-f \sum_{i=1}^{m}\left\langle\mathrm{D}_{e_{i}}(f n), e_{i}\right\rangle=f^{2} H,
$$

where $H$ is the mean curvature of $\partial \mathrm{M}$. Since $\partial \mathrm{M}$ is convex by our hypothesis, $H>0$, so the boundary term in (14) is positive, so $\mathrm{V} \equiv 0$ on $\mathrm{M}$. This proves the existence and uniqueness of $\mathrm{V}$ in the lemma.

The deformation induced by $2 \delta_{g}^{*} \mathrm{~V}$ on $\partial \mathrm{M}$ is easy to compute: for $m \in \partial \mathrm{M}$ and $x, y \in \mathrm{T}_{m} \partial \mathrm{M}$ :

$2\left(\delta_{g}^{*} \mathrm{~V}\right)(x, y)=g\left(\mathrm{D}_{x} \mathrm{~V}, y\right)+g\left(\mathrm{D}_{y} \mathrm{~V}, x\right)=g\left(\mathrm{D}_{x}(f n), y\right)+g\left(\mathrm{D}_{y}(f n), x\right)=-2 f \Pi(x, y)$.

The smoothness and tameness of $\mathcal{F}$ is a consequence of its definition by solution of some elliptic PDEs; we leave it to the reader. Finally, the injectivity of $\mathcal{F}$ is obvious, because if $\mathcal{F}\left(\mathrm{G}^{\prime}\right)=0$, then $\mathrm{G}^{\prime}$ corresponds to a metric variation which is zero inside $\mathrm{M}$, and to a null deformation of the boundary, so that $\mathrm{G}^{\prime}$ is trivial. $\mathcal{F}$ is therefore an isomorphism since equation (11) has index 0 .

To check that $\mathcal{F}$ is equivariant, let $u \in \mathcal{D}_{0}$. Then:

$$
\begin{aligned}
& \left(2 \delta_{u^{*} g}+d \operatorname{tr}_{u^{*} g}\right)\left(u^{*} \mathrm{G}^{\prime}+2 \delta_{u^{*} g}^{*} \mathrm{~V}\right)=\left(2 \delta_{u^{*} g}+d \operatorname{tr}_{u^{*} g}\right)\left(u^{*}\left(\mathrm{G}^{\prime}+2 \delta_{g}^{*} \mathrm{~V}\right)\right)= \\
& =u^{*}\left(\left(2 \delta_{g}+d \operatorname{tr}_{g}\right)\left(\mathrm{G}^{\prime}+2 \delta_{g}^{*} \mathrm{~V}\right)\right)=0 .
\end{aligned}
$$


Moreover, on $\partial \mathrm{M}$ :

$$
\operatorname{tr}_{u^{*} g}\left(u^{*} \mathrm{G}^{\prime}\right)=u^{*}\left(\operatorname{tr}_{g} \mathrm{G}^{\prime}\right)=0
$$

Finally, its definition shows that $\mathrm{F}$ does not depend on $u$, so that:

$$
\mathcal{F}_{u^{*} g}\left(u^{*} \mathrm{G}^{\prime}\right)=\left(u^{*} \mathrm{G}, f\right),
$$

which is the equivariance property we need.

If $\mathrm{K}_{0}=0$, it is enough to consider metrics which are close to the metric on the unit ball in $\mathbf{R}^{n+1}$. Lemma 1 can then be proved just as in the case $K_{0}=-1$, but the crucial point is now that the Ricci curvature of the metric in the ball remains smaller than the first eigenvalue of the Laplacian acting on vector fields (for Dirichlet boundary values). The same applies to lemma 2.

\section{Ellipticity of a boundary value problem}

This section contains the proof of the following lemma of section 2:

Lemma 3. The system:

$$
\left\{\begin{array}{rr}
\mathrm{D}_{g}^{*} \mathrm{D}_{g} \mathrm{G}-2 \stackrel{\circ}{\mathrm{R}}_{g} \mathrm{G}=\mathrm{R} & \text { on } \mathrm{M} \\
\operatorname{tr}_{g} \mathrm{G}=0 \\
\left(2 \delta_{g}+d \operatorname{tr}_{g}\right) \mathrm{G}=0 \\
\partial \mathrm{G} \equiv H \bmod \mathrm{II}
\end{array}\right\} \quad \text { on } \partial \mathrm{M}
$$

is an elliptic boundary problem on $\mathrm{M}$ with index 0 .

Consider, for $t \in[0,1]$, the deformed problem:

$$
\left\{\begin{aligned}
& \mathrm{D}_{g}^{*} \mathrm{D}_{g} \mathrm{G}-2 \stackrel{\circ}{\mathrm{R}} \mathrm{G}=\mathrm{R} \text { on } \mathrm{M} \\
& \operatorname{tr}_{g}(\mathrm{G})=0 \\
&\left(2 \delta_{g}+(1-t) d \operatorname{tr}_{g}\right) \mathrm{G}=0 \\
& \partial \mathrm{G}-(1-t) H \| \mathrm{II}
\end{aligned}\right\} \quad \text { on } \partial \mathrm{M}
$$

For $t=0,(16)$ is the same as (15), while, for $t=1$, it becomes:

$$
\left\{\begin{array}{rr}
\mathrm{D}_{g}^{*} \mathrm{D}_{g} \mathrm{G}-2 \stackrel{\circ}{\mathrm{R}}_{g} \mathrm{G}=\mathrm{R} & \text { on } \mathrm{M} \\
\operatorname{tr}_{g} \mathrm{G}=0 \\
\delta_{g} \mathrm{G}=0 \\
\partial \mathrm{G} \| \mathrm{II}
\end{array}\right\} \quad \text { on } \partial \mathrm{M}
$$

Then consider the further deformation: 


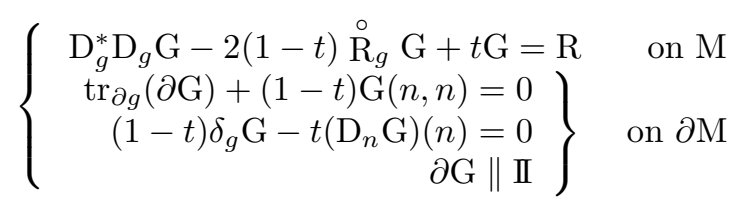

For $t=0,(18)$ is the same as (17), and, for $t=1$, it becomes:

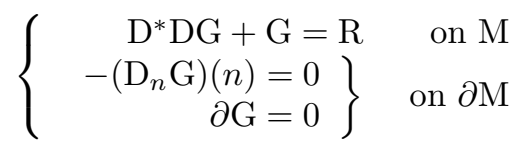

because $\partial \mathrm{G}$ has to be parallel to II and traceless, so it must vanish. Then:

Proposition 7. For all $t \in[0,1],(16)$ is elliptic.

Proposition 8. For all $t \in[0,1]$, (18) is elliptic.

Proposition 9. The problem (19) has a unique solution for $\mathrm{R} \in \mathbf{M}_{g}$.

By proposition 9, (19) is elliptic with index 0; then by propositions 7 and 11 and the invariance of the index, (15) is also elliptic with index 0 , and lemma 3 follows.

Proof of proposition 7: Since ellipticity is invariant under diffeomorphism, we can work in a chart sending $\partial \mathrm{M}$ to $\mathbf{R}^{m} \subset \mathbf{R}^{m+1}$, and such that, at the image of $x$ (which we still call $x$ ) $\mathbb{I}$ is diagonal with (positive) eigenvalues $\mathbb{I}_{1}, \cdots, \mathbb{I}_{m}$.

Ellipticity only depends on the principal symbols of the relevant operators, so we can remove all the terms which do not appear in the principal symbols of either the operator on $\mathrm{M}$ or the boundary conditions, and we are left with the following problem, where $\partial_{i}$ is the derivation with respect to the $i^{t h}$ coordinate:

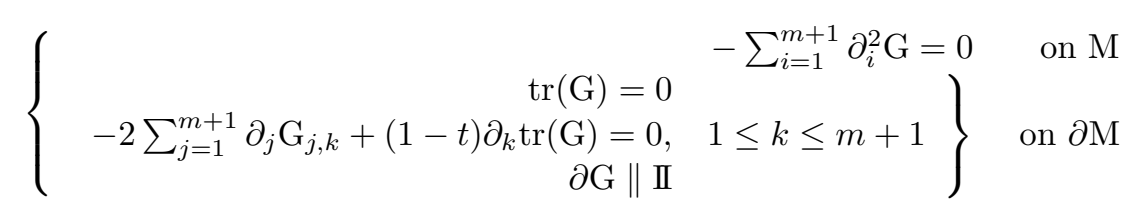

The principal symbol of (20) on $\mathrm{M}$ is, for $y \in \mathbf{R}_{+}^{m+1}$ :

$$
\operatorname{Psym}\left(-\sum_{i=1}^{m+1} \partial_{i}^{2}\right)(y, v) \mathrm{G}=\|v\|^{2} \mathrm{G}
$$

so the space of bounded solutions of the relevant ODE for $x \in \mathbf{R}^{m}$ and $\tilde{v} \in$ $\mathrm{T}_{x} \mathbf{R}^{m}, \tilde{v} \neq 0$, is the following space of functions from $\mathbf{R}_{+}$to the space of $(m+1) \times(m+1)$ symmetric matrices:

$$
\mathcal{M}_{x, \tilde{v}}^{+}=\left\{\left(\mathrm{G}_{j, k}(s)\right)_{1 \leq j, k \leq m+1} \mid \mathrm{G}_{j, k}(s)=\mathrm{G}_{j, k}^{0} e^{-\|\tilde{v}\| s}\right\}
$$


The principal symbols of the boundary conditions are:

$$
\begin{gathered}
\operatorname{tr}(\mathrm{G}) \\
2\|\tilde{v}\| \mathrm{G}_{m+1, k}-2 i \sum_{j=1}^{m} \tilde{v}_{j} \mathrm{G}_{j, k}+(1-t) i \tilde{v}_{k} \operatorname{tr}(\mathrm{G}), 1 \leq k \leq m \\
2\|\tilde{v}\| \mathrm{G}_{m+1, m+1}-2 i \sum_{j=1}^{m} \tilde{v}_{j} \mathrm{G}_{j, m+1}-(1-t)\|\tilde{v}\| \operatorname{tr}(\mathrm{G}) \\
\pi_{\mathbb{I I}^{\perp}}(\partial \mathrm{G})
\end{gathered}
$$

where $\pi_{\mathbb{I I} \perp}$ is the projection to the hyperplane orthogonal to II. We need to prove that the operator $\mathcal{M}_{x, \tilde{v}}^{+} \rightarrow \mathbf{C}^{m(m+1) / 2}$ defined by those principal symbols is injective.

If $\left(\mathrm{G}_{j, k}(s)\right)_{1 \leq j, k \leq m+1} \in \mathcal{M}_{x, \tilde{v}}^{+}$, then the boundary conditions in (21) are 0 if and only if there exists $u$ such that:

$$
\left\{\begin{array}{cc}
\operatorname{tr}(\mathrm{G})=0 & \\
2\|\tilde{v}\| \mathrm{G}_{m+1, k}-2 i \sum_{j=1}^{m} \tilde{v}_{j} \mathrm{G}_{j, k}+(1-t) i \tilde{v}_{k} \operatorname{tr}(\mathrm{G})=0, & 1 \leq k \leq m \\
2\|\tilde{v}\| \mathrm{G}_{m+1, m+1}-2 i \sum_{j=1}^{m} \tilde{v}_{j} \mathrm{G}_{j, m+1}-(1-t)\|\tilde{v}\| \operatorname{tr}(\mathrm{G})=0 & \\
\mathrm{G}_{j, k}=0, & 1 \leq j \neq k \leq m \\
\mathrm{G}_{k, k}=u \Pi_{k}, & 1 \leq k \leq m
\end{array} .\right.
$$

Then $u=-\frac{\mathrm{G}_{m+1, m+1}}{\operatorname{tr}(\mathbb{I})}$, and:

$$
\left\{\begin{array}{cc}
\|\tilde{v}\| \mathrm{G}_{m+1, k}-i \tilde{v}_{k} \mathrm{G}_{k, k}=0 & 1 \leq k \leq m \\
\|\tilde{v}\| \mathrm{G}_{m+1, m+1}-i \sum_{j=1}^{m} \tilde{v}_{j} \mathrm{G}_{j, m+1}=0 & 1 \leq j \neq k \leq m \\
\mathrm{G}_{j, k}=0 & 1 \leq k \leq m \\
\mathrm{G}_{k, k}=-\frac{\mathrm{G}_{m+1, m+1}}{\operatorname{tr}(\mathrm{I})} \mathbb{I}_{k} &
\end{array}\right.
$$

Replacing the second equation in the first and using the last equation of (23) shows that:

$$
\|\tilde{v}\|^{2} \operatorname{tr}(\mathbb{I}) \mathrm{G}_{m+1, m+1}=\mathbb{I}(\tilde{v}, \tilde{v}) \mathrm{G}_{m+1, m+1} .
$$

But II is positive definite, so that $\operatorname{tr}(\mathbb{I})\|\tilde{v}\|^{2}>\mathbb{I}(\tilde{v}, \tilde{v})$, and $\mathrm{G}_{m+1, m+1}=0$. It is then easy to check from $(23)$ that $\left(\mathrm{G}_{i, j}\right)_{1 \leq i, j \leq m+1}=0$, as needed.

Proof of proposition 8: As above, we use a coordinate system and remove the terms which do not appear in the principal symbol. We are left with:

$$
\begin{array}{rr}
\operatorname{tr}(\partial \mathrm{G})+(1-t) \mathrm{G}_{m+1, m+1}=0 & -\sum_{i=1}^{m+1} \partial_{i}^{2} \mathrm{G}=0 \\
-\partial_{m+1} \mathrm{G}_{m+1, k}-(1-t) \sum_{j=1}^{m} \partial_{j} \mathrm{G}_{j, k}=0, & 1 \leq k \leq m+1 \\
\partial \mathrm{G} \| \mathrm{II} & \text { on } \mathrm{M} \\
& \text { on } \partial \mathrm{M}
\end{array}
$$

which is elliptic if and only if (18) is (and has the same index).

The space $\mathcal{M}_{x, \tilde{v}}^{+}$is the same as above in the proof of proposition 7 , and we have to check again that the linear operator from $\mathcal{M}_{x, \tilde{v}}^{+}$to $\mathbf{C}^{m(m+1) / 2}$ defined by the principal symbols of the boundary conditions is injective. 
The principal symbols of the boundary conditions are now:

$$
\begin{gathered}
\sum_{j=1}^{m} \mathrm{G}_{j, j}+(1-t) \mathrm{G}_{m+1, m+1} \\
\|\tilde{v}\| \mathrm{G}_{m+1, k}-i(1-t) \sum_{j=1}^{m} \tilde{v}_{j} \mathrm{G}_{j, k}, 1 \leq k \leq m+1 \\
\pi_{\mathbb{I}^{\perp}}(\partial \mathrm{G})
\end{gathered}
$$

If $\left(\mathrm{G}_{j, k}(s)\right)_{1 \leq j, k \leq m+1} \in \mathcal{M}_{x, \tilde{v}}^{+}$, then the condition that the boundary conditions in (25) are 0 is that there exists $u$ such that:

$$
\left\{\begin{array}{cc}
\sum_{j=1}^{m} \mathrm{G}_{j, j}+(1-t) \mathrm{G}_{m+1, m+1}=0 & \\
\|\tilde{v}\| \mathrm{G}_{m+1, k}-i(1-t) \sum_{j=1}^{m} \tilde{v}_{j} \mathrm{G}_{j, k}=0 & 1 \leq k \leq m+1 \\
\mathrm{G}_{j, k}=0 & 1 \leq j \neq k \leq m \\
\mathrm{G}_{k, k}=u \mathbb{I}_{k} & 1 \leq k \leq m
\end{array}\right.
$$

Then $u=-\frac{(1-t) \mathrm{G}_{m+1, m+1}}{\operatorname{tr}(\mathbb{I})}$, and:

$$
\left\{\begin{array}{cc}
\|\tilde{v}\| \mathrm{G}_{m+1, k}-i(1-t) \tilde{v}_{k} \mathrm{G}_{k, k}=0 & 1 \leq k \leq m \\
\|\tilde{v}\| \mathrm{G}_{m+1, m+1}-i(1-t) \sum_{j=1}^{m} \tilde{v}_{j} \mathrm{G}_{j, m+1}=0 & \\
\mathrm{G}_{j, k}=0 & 1 \leq j \neq k \leq m \\
\mathrm{G}_{k, k}=-\frac{(1-t) \mathrm{G}_{m+1, m+1}}{\operatorname{tr}(\mathrm{II})} \Pi_{k} & 1 \leq k \leq m
\end{array}\right.
$$

Replacing the last equation in the first and using the second equation of (27) shows that:

$$
\|\tilde{v}\|^{2} \operatorname{tr}(\Pi) \mathrm{G}_{m+1, m+1}=(1-t)^{3} \mathrm{G}_{m+1, m+1} \Pi(\tilde{v}, \tilde{v})
$$

But again $\operatorname{tr}(\mathbb{I})\|\tilde{v}\|^{2}>\Pi(\tilde{v}, \tilde{v})$ so that $\mathrm{G}_{m+1, m+1}=0$, and, from $(26), \mathrm{G}=0$ as needed.

Proof of proposition 9: The proof is by minimization again, this time of the functional:

$$
\mathrm{F}(\mathrm{G})=\int_{\mathrm{M}}\|\mathrm{DG}\|^{2}+\|\mathrm{G}\|^{2}-2\langle\mathrm{R}, \mathrm{G}\rangle d v
$$

which is convex and coercive on the vector space of $H^{1}$ sections of $\mathbf{M}_{g}$ which verify the boundary conditions.

\section{Rigidity}

We prove in this section the following lemma of section 2 :

Lemma 4. If $r\left(g_{0}\right)=0$ and $\partial \mathrm{M}$ is strictly convex and umbilical for $g_{0}$, and if $g_{0}$ satisfies (1) (or (2)), then the only solution of (6) for $\mathrm{R} \equiv 0$ and $H \equiv 0$ is $\mathrm{G} \equiv 0$.

We suppose that $g_{0}$ satisfies the hypothesis of the lemma and that $\mathrm{G} \in \mathbf{M}_{g_{0}, \delta}$ is a variation of $g_{0}$ such that $\partial_{\mathrm{I}} \mathrm{G}=0$ which is a solution of (6) with $\mathrm{R}=0$. 
Taking the trace of the first equation of (6) shows that:

$$
\Delta_{g_{0}} \operatorname{tr}_{g_{0}} \mathrm{G}=m \mathrm{~K}_{0} \operatorname{tr}_{g_{0}} \mathrm{G}
$$

Integrating by parts shows that, since $\operatorname{tr}(\mathrm{G})=0$ on $\partial \mathrm{M}$ :

$$
\int_{\mathrm{M}}\left\langle\mathrm{G}, \Delta_{g_{0}} \operatorname{tr}_{g_{0}} \mathrm{G}-m \mathrm{~K}_{0} \operatorname{tr}_{g_{0}} \mathrm{G}\right\rangle d v=\int_{\mathrm{M}}\left\|\mathrm{D}_{g_{0}} \mathrm{G}\right\|^{2}-m \mathrm{~K}_{0}\|\mathrm{G}\| d v=0
$$

and both terms are non-negative, so that $\operatorname{tr}_{g_{0}} \mathrm{G} \equiv 0$ on $\mathrm{M}$. Moreover, proposition 6 shows that $\left(2 \delta_{g_{0}}+d \operatorname{tr}_{g_{0}}\right) \mathrm{G}=0$ on $\mathrm{M}$. But $\operatorname{tr}_{g_{0}} \mathrm{G}=0$, so that $\delta_{g_{0}} \mathrm{G}=0$ on M.

Since $H=0$ and since $\partial \mathrm{M}$ is umbilical, there exists $u: \partial \mathrm{M} \rightarrow \mathbf{R}$ such that:

$$
\forall a \in \partial \mathrm{M}, \forall x, y \in \mathrm{T}_{a} \partial \mathrm{M}, \mathrm{G}(x, y)=u(a) g_{0}(x, y)
$$

and, since $\operatorname{tr}_{g_{0}} \mathrm{G}=0, \mathrm{G}(n, n)=-m u(a)$.

For $m \in \partial \mathrm{M}$, we call $b$ the unique vector in $\mathrm{T}_{m} \partial \mathrm{M}$ such that:

$$
\forall x \in \mathrm{T}_{m} \partial \mathrm{M}, \mathrm{G}(x, n)=g_{0}(x, b)
$$

and also the dual 1 -form on $\partial \mathrm{M}$. We overline all the natural objects on $\partial \mathrm{M}$ to distinguish them from the same objects on $\mathrm{M}$, and we choose an orthonormal moving frame $\left(e_{i}\right)_{1 \leq i \leq m}$ on $\partial \mathrm{M}$. To keep readable notations, we apply an implicit summation to repeated indices, and consider $\mathrm{G}$ both as a symmetric 2 -form and as a linear morphism $\mathrm{TM} \rightarrow \mathrm{TM}$.

The proof rests on two Weitzenböck formulas. The first is:

$$
\begin{gathered}
\int_{\mathrm{M}}\left\|\mathrm{D}_{g_{0}} \mathrm{G}\right\|^{2} d v=\int_{\mathrm{M}} 2\left\langle\stackrel{\circ}{\mathrm{R}}_{g_{0}} \mathrm{G}, \mathrm{G}\right\rangle d v+(m+3) \int_{\partial \mathrm{M}} u(\bar{\delta} b) d a- \\
-\int_{\partial \mathrm{M}}(m+1)^{2} H u^{2}+2 H\|b\|^{2}+2 \mathrm{I}(b, b) d a
\end{gathered}
$$

For the second, consider the operator of exterior differentiation on $\mathrm{T}^{*} \mathrm{M}$-valued forms on $\mathrm{M}$ :

$$
d^{\mathrm{D}}: \mathrm{C}^{\infty}\left(\Lambda^{k} \mathrm{~T}^{*} \mathrm{M} \otimes \mathrm{T}^{*} \mathrm{M}\right) \rightarrow \mathrm{C}^{\infty}\left(\Lambda^{k+1} \mathrm{~T}^{*} \mathrm{M} \otimes \mathrm{T}^{*} \mathrm{M}\right)
$$

and call $\delta^{\mathrm{D}}$ its adjoint. A straightforward computations shows that $\delta^{\mathrm{D}}$ acts as $\delta_{g_{0}}$ on symmetric 2 -forms, when they are considered as 1 -forms with values in $\mathrm{T}^{*} \mathrm{M}$. Therefore, $\delta^{\mathrm{D}} \mathrm{G}=0$. But (see [Bes87], 12.69):

$$
\left(\delta^{\mathrm{D}} d^{\mathrm{D}}+d^{\mathrm{D}} \delta^{\mathrm{D}}\right) \mathrm{G}=\mathrm{D}_{g_{0}}^{*} \mathrm{D}_{g_{0}} \mathrm{G}-\stackrel{\circ}{\mathrm{R}}_{g_{0}} \mathrm{G}+\mathrm{G} \circ \operatorname{ric}_{g_{0}}
$$

We will use this formula to obtain:

$$
\begin{aligned}
& \int_{\mathrm{M}}\left\|d^{\mathrm{D}} \mathrm{G}\right\|^{2} d v=\int_{\mathrm{M}}\left\langle\stackrel{\circ}{\mathrm{R}}_{g_{0}} \mathrm{G}, \mathrm{G}\right\rangle+m \mathrm{~K}_{0}\langle\mathrm{G}, \mathrm{G}\rangle d v- \\
& -(m-1) \int_{\partial \mathrm{M}} u(\bar{\delta} b) d a-\int_{\partial \mathrm{M}}\left(H\|b\|^{2}-\Pi(b, b)\right) d a
\end{aligned}
$$


The proof of lemma 4 follows; by taking a linear combination of equations (29) and (31) with coefficients $m-1$ and $m+3$ respectively, we find that:

$$
\begin{gathered}
\int_{\mathrm{M}}(m-1)\left\|\mathrm{D}_{g_{0}} \mathrm{G}\right\|^{2}+(m+3)\left\|d^{\mathrm{D}} \mathrm{G}\right\|^{2} d v \leq \\
\leq \int_{\mathrm{M}}\left\langle(3 m+1) \stackrel{\circ}{\mathrm{R}}_{g_{0}} \mathrm{G}, \mathrm{G}\right\rangle+\mathrm{K}_{0} m(m+3)\langle\mathrm{G}, \mathrm{G}\rangle d v
\end{gathered}
$$

because the boundary term is non-positive, while it is clear that the left-hand side is non-negative. Moreover, with the hypothesis of lemma 4, the right-hand side is non-positive (this is why we need equation 1 or equation (2)). Both sides are therefore zero. If $K_{0}=-1$, this already shows that $\mathrm{G} \equiv 0$.

If $\mathrm{K}_{0}=0$, the boundary term has to be zero too:

$\int_{\partial \mathrm{M}}(m-1)\left((m+1)^{2} H u^{2}+2 H\|b\|^{2}+\Pi(b, b)\right)+(m+3)\left(H\|b\|^{2}-\Pi(b, b)\right) d a=0$

so $u=b=0$ on $\partial \mathrm{M}$, therefore $\mathrm{G} \equiv 0$ on $\partial \mathrm{M}$, and so $\mathrm{G} \equiv 0$ on $\mathrm{M}$ because $\mathrm{DG} \equiv 0$.

To prove (29), we use that $\mathrm{D}_{g_{0}}^{*} \mathrm{D}_{g_{0}} \mathrm{G}=2 \stackrel{\circ}{\mathrm{R}}_{g_{0}} \mathrm{G}$ (from (6)) to find that:

$$
\begin{aligned}
\int_{M}\left\|\mathrm{D}_{g_{0}} \mathrm{G}\right\|^{2} d v & =\int_{\mathrm{M}}\left\langle\mathrm{D}_{g_{0}}^{*} \mathrm{D}_{g_{0}} \mathrm{G}, \mathrm{G}\right\rangle-\int_{\partial \mathrm{M}}\left\langle\mathrm{D}_{n} \mathrm{G}, \mathrm{G}\right\rangle d a \\
& =\int_{\mathrm{M}}\left\langle 2 \stackrel{\circ}{\mathrm{R}}_{g_{0}} \mathrm{G}, \mathrm{G}\right\rangle-\mathrm{J}
\end{aligned}
$$

Then:

$$
\begin{aligned}
\mathrm{J} & =\int_{\partial \mathrm{M}} g_{0}\left(\left(\mathrm{D}_{n} \mathrm{G}\right)\left(e_{i}\right), \mathrm{Ge}_{i}\right)+g_{0}\left(\left(\mathrm{D}_{n} \mathrm{G}\right)(n), \mathrm{G} n\right) d a \\
& =\int_{\partial \mathrm{M}} u g_{0}\left(\left(\mathrm{D}_{n} \mathrm{G}\right)\left(e_{i}\right), e_{i}\right)+b_{i} g_{0}\left(\left(\mathrm{D}_{n} \mathrm{G}\right)\left(e_{i}\right), n\right)+g_{0}\left(\left(\mathrm{D}_{n} \mathrm{G}\right)(n), \mathrm{G} n\right) d a
\end{aligned}
$$

But:

$$
g_{0}\left(\left(\mathrm{D}_{n} \mathrm{G}\right)\left(e_{i}\right), e_{i}\right)+g_{0}\left(\left(\mathrm{D}_{n} \mathrm{G}\right) n, n\right)=d\left(\operatorname{tr}_{g_{0}} \mathrm{G}\right)(n)=0
$$

and $\mathrm{G}$ is symmetric, so that:

$$
g_{0}\left(\left(\mathrm{D}_{n} \mathrm{G}\right)\left(e_{i}\right), n\right)=g_{0}\left(\left(\mathrm{D}_{n} \mathrm{G}\right)(n), e_{i}\right)
$$

Therefore:

$$
\begin{aligned}
\mathrm{J} & =\int_{\partial \mathrm{M}} g_{0}\left(\left(\mathrm{D}_{n} \mathrm{G}\right)(n), b-u n+\mathrm{G} n\right) d a \\
& =\int_{\partial \mathrm{M}} g_{0}\left(\left(\mathrm{D}_{n} \mathrm{G}\right)(n), 2 b-(m+1) u n\right) d a
\end{aligned}
$$

Now

$$
\delta_{g_{0}} \mathrm{G}=0=\left(\mathrm{D}_{n} \mathrm{G}\right)(n)+\left(\mathrm{D}_{e_{i}} \mathrm{G}\right) e_{i}
$$


so

$$
\mathrm{J}=\int_{\partial \mathrm{M}} g_{0}\left(\left(\mathrm{D}_{e_{i}} \mathrm{G}\right)\left(e_{i}\right),(m+1) u n-2 b\right) d a
$$

But:

$$
g_{0}\left(\left(\mathrm{D}_{e_{i}} \mathrm{G}\right)\left(e_{i}\right), b\right)=e_{i} \cdot \mathrm{G}\left(e_{i}, b\right)-\mathrm{G}\left(\mathrm{D}_{e_{i}} e_{i}, b\right)-\mathrm{G}\left(e_{i}, \mathrm{D}_{e_{i}} b\right)
$$

and $\mathrm{D}_{x} y=\overline{\mathrm{D}}_{x} y+\mathbb{\Pi}(x, y) n$, so that:

$$
\begin{gathered}
g_{0}\left(\left(\mathrm{D}_{e_{i}} \mathrm{G}\right)\left(e_{i}\right), b\right)=e_{i} \cdot\left(u b\left(e_{i}\right)\right)-u b\left(\overline{\mathrm{D}}_{e_{i}} e_{i}\right)-\Pi\left(e_{i}, e_{i}\right) \mathrm{G}(b, n)- \\
-\mathrm{G}\left(e_{i}, \overline{\mathrm{D}}_{e_{i}} b\right)-\Pi\left(e_{i}, b\right) \mathrm{G}\left(e_{i}, n\right)
\end{gathered}
$$

so

$$
\begin{aligned}
g_{0}\left(\left(\mathrm{D}_{e_{i}} \mathrm{G}\right)\left(e_{i}\right), b\right) & =b\left(e_{i}\right) \overline{\mathrm{D}} u\left(e_{i}\right)-H\|b\|^{2}-\Pi(b, b) \\
& =g_{0}(b, \overline{\mathrm{D}} u)-H\|b\|^{2}-\Pi(b, b)
\end{aligned}
$$

On the other hand:

$$
\begin{aligned}
g_{0}\left(\left(\mathrm{D}_{e_{i}} \mathrm{G}\right)\left(e_{i}\right), n\right) & =e_{i} \cdot \mathrm{G}\left(e_{i}, n\right)-\mathrm{G}\left(\mathrm{D}_{e_{i}} e_{i}, n\right)-\mathrm{G}\left(e_{i}, \mathrm{D}_{e_{i}} n\right) \\
& =e_{i} \cdot b\left(e_{i}\right)-b\left(\overline{\mathrm{D}}_{e_{i}} e_{i}\right)+\mathrm{I}\left(e_{i}, e_{i}\right) m u+\mathrm{G}\left(e_{i}, \mathrm{~B} e_{i}\right) \\
& =\left(\overline{\mathrm{D}}_{e_{i}} b\right)\left(e_{i}\right)+m H u+H u \\
& =-(\bar{\delta} b)+(m+1) H u
\end{aligned}
$$

Replacing (33) and (34) in (32) gives:

$$
\mathrm{J}=\int_{\partial \mathrm{M}}-(m+1) u(\bar{\delta} b)+(m+1)^{2} H u^{2}-2 g_{0}(b, \overline{\mathrm{D}} u)+2 H\|b\|^{2}+2 \Pi(b, b) d a
$$

from which (29) follows by integration by parts of $g_{0}(b, \overline{\mathrm{D}} u)$.

The proof of (31) is similar:

$$
\begin{aligned}
\int_{\mathrm{M}}\left\|d^{\mathrm{D}} \mathrm{G}\right\|^{2} d v & =\int_{\mathrm{M}}\left\langle\delta^{\mathrm{D}} d^{\mathrm{D}} \mathrm{G}, \mathrm{G}\right\rangle d v-\int_{\partial \mathrm{M}}\left\langle\left(d^{\mathrm{D}} \mathrm{G}\right)(n, .), \mathrm{G}\right\rangle d a \\
& =\int_{\mathrm{M}}\left\langle\delta^{\mathrm{D}} d^{\mathrm{D}} \mathrm{G}+d^{\mathrm{D}} \delta^{\mathrm{D}} \mathrm{G}, \mathrm{G}\right\rangle d v-\int_{\partial \mathrm{M}}\left\langle\left(d^{\mathrm{D}} \mathrm{G}\right)(n, .), \mathrm{G}\right\rangle d a \\
& =\int_{\mathrm{M}}\left\langle\stackrel{\circ}{\mathrm{R}}_{g_{0}} \mathrm{G}+m \mathrm{~K}_{0} \mathrm{G}, \mathrm{G}\right\rangle d v-\mathrm{J}^{\prime}
\end{aligned}
$$


from (30) and (6), with:

$$
\begin{aligned}
\mathrm{J}^{\prime} & =\int_{\partial \mathrm{M}} g_{0}\left(\left(d^{\mathrm{D}} \mathrm{G}\right)\left(n, e_{i}\right), \mathrm{G} e_{i}\right) d a \\
& =\int_{\partial \mathrm{M}} g_{0}\left(\left(\mathrm{D}_{n} \mathrm{G}\right)\left(e_{i}\right)-\left(\mathrm{D}_{e_{i}} \mathrm{G}\right)(n), u e_{i}+b_{i} n\right) d a \\
& =\int_{\partial \mathrm{M}} u\left(\mathrm{D}_{n} \mathrm{G}\right)\left(e_{i}, e_{i}\right)+b_{i}\left(\mathrm{D}_{n} \mathrm{G}\right)\left(e_{i}, n\right)-u\left(\mathrm{D}_{e_{i}} \mathrm{G}\right)\left(n, e_{i}\right)-b_{i}\left(\mathrm{D}_{e_{i}} \mathrm{G}\right)(n, n) d a \\
& =\int_{\partial \mathrm{M}}-u\left(\mathrm{D}_{n} \mathrm{G}\right)(n, n)-b_{i}\left(\mathrm{D}_{e_{j}} \mathrm{G}\right)\left(e_{i}, e_{j}\right)-u\left(\mathrm{D}_{e_{i}} \mathrm{G}\right)\left(n, e_{i}\right)-b_{i}\left(\mathrm{D}_{e_{i}} \mathrm{G}\right)(n, n) d a \\
& =\int_{\partial \mathrm{M}}-\left(\mathrm{D}_{e_{j}} \mathrm{G}\right)\left(b, e_{j}\right)-\left(\mathrm{D}_{b} \mathrm{G}\right)(n, n) d a \\
& =\int_{\partial \mathrm{M}}-e_{j} \cdot\left(u b\left(e_{j}\right)\right)+\mathrm{G}\left(\mathrm{D}_{e_{j}} b, e_{j}\right)+\mathrm{G}\left(b, \mathrm{D}_{e_{j}} e_{j}\right)+m d u(b)+2 \mathrm{G}\left(\mathrm{D}_{b} n, n\right) d a \\
& =\int_{\partial \mathrm{M}}-d u(b)+\mathbb{I}\left(e_{i}, e_{i}\right) \mathrm{G}(b, n)+\mathbb{I}\left(e_{i}, b\right) \mathrm{G}\left(n, e_{i}\right)+m d u(b)-2 \mathrm{G}(\mathrm{B} b, n) d a \\
& =\int_{\partial \mathrm{M}}(m-1) d u(b)+H\|b\|^{2}+\mathbb{I}(b, b)-2 \mathbb{I}(b, b) d a \\
& =\int_{\partial \mathrm{M}}(m-1) d u(b)+H\|b\|^{2}-\mathbb{I}(b, b) d a
\end{aligned}
$$

and (31) follows. This ends the proof of lemma 4.

\section{Bilinear error terms}

We finish here the proof of theorem 6 by giving the proof of lemma 6 . First, we will prove proposition 6 . For this, we will use an analogous, non-linear result. For $g \in \mathcal{M}$, let:

$$
\begin{aligned}
\Phi_{g}: \mathcal{M} & \rightarrow \mathcal{M} \\
h & \mapsto r i c_{h}-m \mathrm{~K}_{0} h+\delta_{h}^{*}\left(2 \delta_{g}+d \operatorname{tr}_{g}\right) h
\end{aligned}
$$

The following proposition is a slight extension of lemma 1.6 of [Biq00]:

Proposition 10. Suppose that ric $_{h} \leq 0$, and that $\Phi_{g}(h)=p$ with $\left(2 \delta_{h}+\right.$ $\left.d \operatorname{tr}_{h}\right) p=0$. If $\left(2 \delta_{g}+d \operatorname{tr}_{g}\right) h=0$ on $\partial \mathrm{M}$, then $\left(2 \delta_{g}+d \operatorname{tr}_{g}\right) h=0$ on $\mathrm{M}$, and ric $_{h}-m \mathrm{~K}_{0} h=p$ on $\mathrm{M}$. 
Taking the trace of the differential Bianchi identity shows that:

$$
2 \delta_{h} \text { ric }_{h}+d \mathrm{~S}_{h}=\left(2 \delta_{h}+d \operatorname{tr}_{h}\right) \text { ric }_{h}=0,
$$

where $\mathrm{S}_{h}$ is the scalar curvature of $h$. But:

$$
\left(2 \delta_{h}+d \operatorname{tr}_{h}\right) \Phi_{g}(h)=\left(2 \delta_{h}+d \operatorname{tr}_{h}\right) p=0,
$$

so that, by definition of $\Phi_{g}$ and (35):

$$
\left(2 \delta_{h} \delta_{h}^{*}-d \delta_{h}\right)\left(2 \delta_{g} h+d \operatorname{tr}_{g} h\right)=0 .
$$

Setting $c=2 \delta_{g} h+d \operatorname{tr}_{g} h$ and using (10) shows that:

$$
\left(\mathrm{D}_{h}^{*} \mathrm{D}_{h}-\mathrm{ric}_{h}\right) c=0 \text {. }
$$

Integrating against $c$ and using that $c=0$ on $\partial \mathrm{M}$ leads to:

$$
\int_{\mathrm{M}}\left\langle\left(\mathrm{D}_{h}^{*} \mathrm{D}_{h}-\operatorname{ric}_{h}\right) c, c\right\rangle d v=\int_{\mathrm{M}}\left\|\mathrm{D}_{h} c\right\|^{2}-\left\langle\operatorname{ric}_{h} c, c\right\rangle d v=0,
$$

and ric $_{h} \leq 0$, so that, (using again that $c=0$ on $\partial \mathrm{M}$ ) $c=0$ on $\mathrm{M}$. It is then obvious from the definition of $\Phi_{g}$ (and the hypothesis that $\Phi_{g}(h)=p$ ) that ric $_{h}-m \mathrm{~K}_{0} h=p$.

The proof of proposition 6 is then a simple consequence of proposition 10 and of its proof; linearizing each step of the proof of proposition 10 gives a proof of proposition 6 .

Proof of lemma 6: Choose $g \in \mathcal{M}$ and $\mathrm{G} \in \mathbf{M}_{g, \delta}$. By lemma 2, $\mathrm{G}=$ $\mathrm{G}_{0}-2 \delta_{g}^{*} \mathrm{~V}$, with $\mathrm{G}_{0} \in \mathbf{M}_{g, \delta, t}$ and $\mathrm{V} \in \mathbf{V}_{\perp}$. We will show (7) first for $\mathrm{G}_{0}$, then for $-2 \delta_{g}^{*} \mathrm{~V}$.

Let $(H, \mathrm{R})=\left(\partial \times \mathrm{D}^{\Gamma} r\right) \mathrm{G}_{0}$, and let $\mathrm{G}_{0}^{\prime}=\mathrm{V}_{g}(H, \mathrm{R}) \in \mathbf{M}_{g, \delta}$. The variation of $r$ associated to $\mathrm{G}_{0}$ is:

$$
r^{\prime}=\mathrm{D}_{g}^{*} \mathrm{D}_{g} \mathrm{G}_{0}-\delta_{g}^{*}\left(2 \delta_{g}+d \operatorname{tr}_{g}\right) \mathrm{G}_{0}-2 \stackrel{\circ}{\mathrm{R}}_{g} \mathrm{G}_{0}=\mathrm{D}_{g}^{*} \mathrm{D}_{g} \mathrm{G}_{0}-2 \stackrel{\circ}{\mathrm{R}}_{g} \mathrm{G}_{0} .
$$

But $r$ is a section of $\mathcal{R}_{g, \delta}$, i.e.:

$$
\left(2 \delta_{g}+d \operatorname{tr}_{g}\right) r=0 .
$$

Linearizing this equality shows that:

$$
\left(2 \delta_{g}+d \operatorname{tr}_{g}\right) r^{\prime}+\left(2 \delta_{g}^{\prime}+d \operatorname{tr}_{g}^{\prime}\right) r(g)=0 .
$$

If $r(g)=0$, this already shows that $r^{\prime} \in \mathcal{R}_{g, \delta}$, so that $\mathrm{R}:=\mathrm{D}_{\mathrm{G}_{0}}^{\Gamma} r:=\Pi_{g}\left(r^{\prime}\right)=$ $r^{\prime} . \mathrm{G}_{0}$ is then a solution of the equation used to define $\mathrm{G}_{0}^{\prime}$, and they satisfy the same boundary conditions, so that $\mathrm{G}_{0}^{\prime}=\mathrm{G}_{0}$ by uniqueness of the solution of (6).

If $r(g) \neq 0$, the same idea shows that $\mathrm{G}_{0}^{\prime}$ is "close" to $\mathrm{G}_{0}$ :

$$
\left(2 \delta_{g}+d \operatorname{tr}_{g}\right) r^{\prime}=-\left(2 \delta_{g}^{\prime}+d \operatorname{tr}_{g}^{\prime}\right) r=q_{1, g}^{l}\left(r(g), \mathrm{G}_{0}\right),
$$


where $q_{1, g}^{l}\left(r(g), \mathrm{G}_{0}\right)$ is smooth, tame and bilinear. Therefore, by equation (4) of proposition 3:

$$
\mathrm{R}-r^{\prime}=q_{2, g}^{l}\left(r(g), \mathrm{G}_{0}\right)
$$

where $q_{2, g}^{l}$ is smooth, tame and bilinear. Then $\mathrm{G}_{0}$ and $\mathrm{G}_{0}^{\prime}$ are solutions respectively of:

$$
\mathrm{D}_{g}^{*} \mathrm{D}_{g} \mathrm{G}_{0}-2 \stackrel{\circ}{\mathrm{R}} \mathrm{G}_{0}=r^{\prime}
$$

and of:

$$
\mathrm{D}_{g}^{*} \mathrm{D}_{g} \mathrm{G}_{0}^{\prime}-2 \stackrel{\circ}{\mathrm{R}}_{g} \mathrm{G}_{0}^{\prime}=\mathrm{R},
$$

with the same boundary conditions. Thus:

$$
\mathrm{G}_{0}-\mathrm{G}_{0}^{\prime}=q_{3, g}^{l}\left(r(g), \mathrm{G}_{0}\right),
$$

where again $q_{3, g}^{l}$ is smooth, tame and bilinear. This shows (7) for $\mathrm{G}_{0}$.

Consider now the term $-2 \delta_{g}^{*} \mathrm{~V}$. Let $\mathrm{V}=f n$ on $\partial \mathrm{M}$. If $r(g)=0$, then:

$$
\left(\partial \times \mathrm{D}^{\Gamma} r\right)\left(-2 \delta_{g}^{*} \mathrm{~V}\right)=(2 f \Pi, 0) .
$$

The solution of $(6)$ for $(H, \mathrm{R})=(2 f \Pi, 0)$ is obviously 0 , because the component of $H$ parallel to II doesn't appear in the equation (6). The definition of $\mathrm{V}_{g}$ then shows that $\mathrm{V}_{g}(2 f \mathrm{II}, 0)=-2 \delta_{g}^{*} \mathrm{~V}$, and this proves $(7)$ for this term when $r(g)=0$.

If $r(g) \neq 0$, the same idea will again prove (7). Then $\partial\left(-2 \delta_{g}^{*} \mathrm{~V}\right)=2 f \Pi$, and the variation of $r$ associated to $-2 \delta_{g}^{*} \mathrm{~V}$ is:

$$
r^{\prime}=-\mathcal{L}_{\mathrm{V}} r=q_{4, g}^{l}(r(g), \mathrm{V}),
$$

where $q_{4, g}^{l}$ is smooth, tame and bilinear. The definition of $\Gamma$ then shows that:

$$
\mathrm{D}_{-2 \delta_{g}^{*} \mathrm{~V}}^{\Gamma} r=q_{5, g}^{l}(r(g), \mathrm{V}),
$$

where $q_{5, g}^{l}$ is also smooth, tame and bilinear. The solution $\mathrm{G}^{\prime}$ of $(6)$ for $(H, \mathrm{R})=$ $\left(2 f \mathrm{II}, \mathrm{D}_{-2 \delta_{g}^{*} \mathrm{~V}}^{\Gamma} r\right)$ is then small, again because the component of $H$ parallel to II does not count:

$$
\mathrm{G}^{\prime}=q_{6, g}^{l}(r(g), \mathrm{V}),
$$

where $q_{6, g}^{l}$ is again smooth, tame and bilinear. So we finally obtain that:

$$
\mathrm{V}_{g} \circ\left(\partial, \mathrm{D}^{\Gamma} r\right)\left(-2 \delta_{g}^{*} \mathrm{~V}\right)=-2 \delta_{g}^{*} \mathrm{~V}+q_{6, g}^{l}(r(g), \mathrm{V}) .
$$

This finishes the proof of (7).

Now choose $(H, \mathrm{R}) \in \mathcal{N}_{\partial g} \times \mathcal{R}_{g, \delta}$. Let $\mathrm{G}=\mathrm{V}_{g}(H, \mathrm{R})$ and $\left(H^{\prime}, \mathrm{R}^{\prime}\right)=(\partial \times$ $\left.\mathrm{D}^{\Gamma} r\right) \mathrm{G}$, and let $f: \partial \mathrm{M} \rightarrow \mathbf{R}$ be the function such that $H-2 f \mathrm{II}$ is trace-free for II on $\partial \mathrm{M}$. By definition of $\mathrm{V}_{g}, \mathrm{G}=\Pi_{g}\left(\mathrm{G}^{\prime}\right)+\mathcal{F}_{g}^{-1}(0, f)$, where $\mathrm{G}^{\prime}$ is the solution of (6). Thus, by definition of $\Pi_{g}$ and of $\mathcal{F}_{g}, \mathrm{G}=\mathrm{G}^{\prime}+2 \delta_{g}^{*} \mathrm{~V}$, where $\mathrm{V} \in \mathbf{V}_{\perp}$. One easily checks that $\partial \Pi_{g}\left(\mathrm{G}^{\prime}\right)=\partial \mathrm{G}^{\prime}$, so that $H^{\prime}=H$. 
The variation of $r$ associated to the variation $\mathrm{G}$ of $g$ is:

$$
r^{\prime}=\mathrm{D}_{g}^{*} \mathrm{D}_{g} \mathrm{G}^{\prime}-\delta_{g}^{*}\left(2 \delta_{g}+d \operatorname{tr}_{g}\right) \mathrm{G}^{\prime}-2 \stackrel{\circ}{\mathrm{R}_{g}} \mathrm{G}^{\prime}+\mathcal{L}_{\mathrm{V}} r,
$$

while, by definition of $\mathrm{G}^{\prime}$ :

$$
\mathrm{D}_{g}^{*} \mathrm{D}_{g} \mathrm{G}^{\prime}-2 \stackrel{\circ}{\mathrm{R}} \mathrm{G} \mathrm{G}^{\prime}=\mathrm{R} .
$$

Consequently:

$$
r^{\prime}=\mathrm{R}-\delta_{g}^{*}\left(2 \delta_{g}+d \operatorname{tr}_{g}\right) \mathrm{G}^{\prime}+\mathcal{L}_{\mathrm{V}} r .
$$

By definition of $\Gamma$ :

$$
\mathrm{R}^{\prime}:=\mathrm{D}_{\mathrm{G}}^{\Gamma} r:=\Pi_{g}\left(r^{\prime}\right)=\mathrm{R}-\delta_{g}^{*}\left(2 \delta_{g}+d \operatorname{tr}_{g}\right) \mathrm{G}^{\prime}+\mathcal{L}_{\mathrm{V}} r+2 \delta_{g}^{*} \mathrm{~W}^{\prime},
$$

where $\mathrm{W}^{\prime}$ is the unique element of $\mathbf{V}_{0}$ such that $\left(2 \delta_{g}+d \operatorname{tr}_{g}\right)\left(\mathrm{D}_{\mathrm{G}}^{\Gamma} r\right)=0$. Since the vector field $\left(2 \delta_{g}+d \operatorname{tr}_{g}\right) \mathrm{G}^{\prime}$ vanishes on $\partial \mathrm{M}$ by (6), it can be incorporated into $\mathrm{W}^{\prime}$, so as to obtain that:

$$
\mathrm{R}^{\prime}:=\mathrm{R}+\mathcal{L}_{\mathrm{V}} r+2 \delta_{g}^{*} \mathrm{~W}
$$

where $\mathrm{W} \in \mathbf{V}_{0}$ is such that:

$$
\left(2 \delta_{g}+d \operatorname{tr}_{g}\right)\left(\mathcal{L}_{\mathrm{V}} r+2 \delta_{g}^{*} \mathrm{~W}\right)=0,
$$

because $\left(2 \delta_{g}+d \operatorname{tr}_{g}\right) \mathrm{R}=0$ by definition of $\mathrm{R}$. That is:

$$
\mathrm{R}^{\prime}=\mathrm{R}+\Pi_{g}\left(\mathcal{L}_{\mathrm{V}} r\right) .
$$

Now it is quite easy to check, from the definition of $\Pi_{g}$, that there exists $\mathrm{C}(g)>0$ such that:

$$
\left\|\mathcal{L}_{\mathrm{V}} r-\Pi_{g}\left(\mathcal{L}_{\mathrm{V}} r\right)\right\| \leq \mathrm{C}(g)\left\|\mathcal{L}_{\mathrm{V}} r\right\|
$$

As a consequence:

$$
\left\|\mathrm{R}^{\prime}-\mathrm{R}\right\| \leq(1+\mathrm{C}(g))\left\|\mathcal{L}_{\mathrm{V}} r\right\|
$$

If $r(g)=0$, then $\mathrm{R}^{\prime}=\mathrm{R}$, and the proof of (8) follows. If $r(g) \neq 0$, then:

$$
\mathrm{R}^{\prime}-\mathrm{R}=q_{1, g}^{r}(r(g), \mathrm{V}),
$$

where $q_{1, g}^{r}$ is smooth, tame and bilinear, so that, by definition of $\mathrm{V}$ :

$$
\mathrm{R}^{\prime}-\mathrm{R}=q_{2, g}^{r}(r(g),(H, \mathrm{R})),
$$

and $q_{2, g}^{r}$ is smooth, tame and bilinear. This proves (8).

\section{References}

[Ale58] A. D. Aleksandrov, Vestnik Leningrad Univ., 13(1), 1958.

[Bes87] A. Besse, Einstein Manifolds, Springer, 1987.

[Biq00] Olivier Biquard, Métriques d'Einstein asymptotiquement symétriques, volume 265 of Astérisque. Société Mathématique de France, 2000. 
[DeT81] D. M. DeTurck, Existence of metrics with prescribed Ricci curvature: local theory. Invent. Math. 65 (1981), 179-207.

[GL91] C. R. Graham and J. M. Lee, Einstein metrics with prescribed conformal infinity on the ball. Adv. Math. 87 (1991), 186-225.

[Ham75] R. S. Hamilton, Harmonic maps of manifolds with boundary. Springer-Verlag, Berlin, 1975. Lecture Notes in Mathematics, Vol. 471.

[Ham82] R. S. Hamilton, The inverse function theorem of Nash and Moser. Bull. Amer. Math. Soc. (N. S.) 7 (1982), 65-222.

[Lab89] F. Labourie, Immersions isométriques elliptiques et courbes pseudo-holomorphes. $J$. Differential Geom. 30 (1989), 395-424.

[Lab92] F. Labourie, Métriques prescrites sur le bord des variétés hyperboliques de dimension 3. J. Differential Geom. 35 (1992), 609-626.

[LS00] F. Labourie and J.-M. Schlenker, Surfaces convexes fuchsiennes dans les espaces lorentziens à courbure constante. Math. Annalen 316 (2000), 465-483.

[Nir53] L. Nirenberg, The Weyl and Minkowski problem in differential geometry in the large. Comm. Pure Appl. Math. 6 (1953), 337-394.

[Pog73] A. V. Pogorelov, Extrinsic Geometry of Convex Surfaces. American Mathematical Society, 1973. Translations of Mathematical Monographs. Vol. 35.

[RS98] I. Rivin, J.-M. Schlenker, The Schläfli formula and Einstein manifolds. Preprint IHES/M/98/26, 1998.

[RS99] I. Rivin, J.-M. Schlenker, The Schläfli formula in Einstein manifolds with boundary. Electronic Research Announcements of the A.M.S. 5 (1999), 18-23.

[Sch95] G. Schwarz, Hodge decomposition - a method for solving boundary value problems. Springer, 1995. (Lectures Notes in Mathematics, 1607).

[Sch96] J.-M. Schlenker, Surfaces convexes dans des espaces lorentziens à courbure constante. Commun. Anal. and Geom. 4 (1996), 285-331.

[Sch98] J.-M. Schlenker, Métriques sur les polyèdres hyperboliques convexes. J. Differential Geom. 48(2) (1998), 323-405.

[She96] Y. Shen, On Ricci deformation of a Riemannian metric on manifold with boundary. Pacific Journal of Math. 173 (1996), 203-221.

[Ye93] R. Ye, Ricci flow, Einstein metrics and space forms. Trans. of the American Math. Soc. 338 (1993), 871-896.

Jean-Marc Schlenker

Laboratoire Emile Picard (UMR CNRS 5580)

Université Paul Sabatier

118 route de Narbonne

F-31062 Toulouse Cedex 4

France

e-mail: schlenker@picard.ups-tlse.fr

(Received: February 12, 1998) 